\title{
Identification of Outliers in Gene Expression Data
}

\author{
A THESIS \\ OF THE REQUIREMENTS \\ for the degree \\ MASTER OF SCIENCE \\ By \\ Md Manzur Rahman Farazi \\ Adviser Dr. Rahmatullah Imon \\ Ball State University \\ Muncie, Indiana \\ May 2015
}

SUBMITTED TO THE GRADUATE SCHOOL IN PARTIAL FULFILLMENT 


\section{Identification of Outliers in Gene Expression Data A THESIS \\ SUBMITTED TO THE GRADUATE SCHOOL IN PARTIAL FULFILLMENT OF THE \\ REQUIREMENTS \\ for the degree \\ MASTER OF SCIENCE \\ By \\ Md Manzur Rahman Farazi}

Committee Approval:

Committee Chairman

Date

Committee Member

Date

Committee Member

Date

Department Head Approval:

Head of Department

Date

Graduate office Check:

Dean of Graduate School

Date

Ball State University

Muncie, Indiana

May 2015 


\section{ACKNOWLEDGEMENTS}

I would like to thank to my advisor, Professor Dr. Rahmatullah Imon, for supporting me during my thesis tenure. Dr. Imon is someone you will instantly love and never forget once you meet him. He is the funniest advisor and one of the smartest people I know. I hope that I could be as lively, enthusiastic, and energetic as Dr. Imon and to someday be able to command an audience as well as he can. He has been supportive and has given me the freedom to pursue various alternatives without objection. He has also provided insightful discussions about the research.

I am also very grateful to Dr. Munni Begum for her advice and knowledge and many insightful discussions and suggestions. I have learned a lot from her class. I also have to thank the members of my MS advisory committee for their helpful career advice and suggestions in general.

I also thank people who were not part of the research group but helped me out, including Morshed Alam, a graduate student in the department of Mathematical Sciences. I am also thankful to the Bangladeshi community who supported me all the times. I also show my thanks to some of my international friends who helped me in various occasions.

It was a tough time without having my family members with me but they constantly supported me throughout my life at Ball State University. Their unconditional mental support was the strength of being able to complete the thesis.

Md Manzur Rahman Farazi 


\section{ABSTRACT}

This work reports the application of techniques that proved useful in analyzing a large gene expression data set. Because it appears likely that genomic instability in cancers can optimize gene expression for cell growth, the differences between normal and tumor expression patterns might help us understand what is being selected for as cancerous tissues evolve. Molecular heterogeneity of cancer, caused by various gene mutations, can yield extensive heterogeneity in gene expression profile in cancer samples. To detect cancer-related genes which are active only in a subset of cancer samples or cancer outliers, recently, Tomlins et al. (2005) argued that traditional analytical methods, for example, a two-sample t-statistic, which search for common activation of genes across a class of cancer samples, will fail to detect cancer genes which show differential expression in a subset of cancer samples or cancer outliers. They developed the "cancer outlier profile analysis" (COPA) method to detect cancer genes with such heterogeneous expression profiles within cancer samples and revealed subtypes of prostate cancer patients defined by recurrent chromosomal aberration. Inspired by the COPA statistic, some authors have proposed other methods for detecting cancer-related genes with cancer outlier profiles in the framework of multiple testing (Tibshirani-2007, Wu-2007, Lian-2008, Wang2010). Such cancer outlier analyses are affected from many problems specially if there is any outlier in the data set then classical measures of location and scale are seriously affected. So the test statistic using these parameters might not be appropriate to detect outliers. In this study, we try to robustify some existing methods. We propose three new techniques Expressed robust tstatistic (ERT), Modified Outlier robust t-statistic (MORT) and Least Sum Square of Ordered Subset Robust t-statistic (LSOSRT) for the identification of outliers. The usefulness of the proposed methods is then investigated by Monte Carlo simulation and real cancer data. 


\section{Table of Contents}

\section{CHAPTER 1}

1.1 Outliers in Statistical Data

1.2 Gene Expression Data

Error! Bookmark not defined.

1.3 The Need for Detection of Outliers in Gene Expression Data 2

1.4 Outline of the Study

CHAPTER 2

GENE AND ITS PATTERN

2.1 Genes

2.2 Gene Expression $\quad 6$

$\begin{array}{lr}2.3 \text { Transcription } & 7\end{array}$

$\begin{array}{lr}2.4 \text { Non-coding RNA Maturation } & 10\end{array}$

2.5 Translation 11

2.6 Folding 13

$\begin{array}{lr}2.7 \text { Translocation } & 14\end{array}$

2.8 Cancer Data Under Study 14

2.9 Patterns of Gene Expression of Tumor and Normal Colon Tissues 15

$\begin{array}{lr}\text { CHAPTER 3 } & 19\end{array}$

IDENTIFICATION OF OUTLIERS $\quad 19$

$\begin{array}{ll}3.1 \text { Outliers } & 19\end{array}$

3.2 Detection of Outliers 21

3.2.1 The Three-sigma Rule $\quad 22$

3.2.2 Grubbs' Test $\quad 22$

3.2.3 Dixon's Q Test 23

3.3 Robust Outlier Detection Methods 24

3.3.1 Robust t like statistic $\quad 25$

3.3.2 Test Based on the Interquartile Range 25

3.3.3 Hampel's Test 25

3.4 Masking and Swamping Effects 26

$\begin{array}{lr}\text { CHAPTER } 4 & 27\end{array}$

IDENTIFICATION OF OUTLIERS IN GENE DATA 27

4.1 Existing Outlier Detection Methods for Gene Expression Data 27

4.1.1 t-statistic 27

4.1.2 Cancer Outliers Profile Analysis (COPA) 28

4.1.3 Outliers Sums (OS) Statistic 28

4.1.4 Outliers Robust t-statistic (ORT) 29

4.1.5 Maximum Ordered Subset t-statistic (MOST) 29

4.1.6 Least Sumsquare of Ordered Subset Variance t-statistic (LSOSS) 30 
4.2 Proposed Outlier Detection Methods 31

4.2.1 Expressed Robust t-statistic (ERT) 31

4.2.2 Modified Outlier Robust t-statistic (MORT) 32

4.2.3 The Least Sum Square of Ordered Subset Robust t-statistic (LSOSRT) 32

4.3 Monte Carlo Comparison of Different Outlier Detection Methods 34

4.4 Comparison of Different Outlier Detection Methods Using Cancer Data 35

$\begin{array}{ll}\text { CHAPTER 5 } & 39\end{array}$

CONCLUSIONS AND AREAS OF FUTURE RESEARCH

5.1 Conclusions $\quad 39$

5.2 Areas of Future Research $\quad 40$

REFERENCES

$\begin{array}{ll}\text { APPENDIX } & 44\end{array}$

$\begin{array}{ll}\text { I. Sample Data } & 44\end{array}$

$\begin{array}{ll}\text { II. R Code } & 45\end{array}$ 


\section{List of Figures}

\section{Chapter 2}

Figure 2.1: An overview of the flow of information from DNA to protein 6

$\begin{array}{ll}\text { Figure 2.2: The RNA sequence } & 7\end{array}$

Figure 2.3: : Simple illustration of exons and introns in pre-mRNA and the formation of mature mRNA by splicing

Figure 2.4: Protein chain $\quad 11$

$\begin{array}{ll}\text { Figure 2.5: Protein folding } & 13\end{array}$

Figure 2.6: a Plot of the Gene Intensities for Normal Samples 16

Figure 2.6: b Plot of the Gene Intensities for Tumor Samples 16

Figure 2.6: c Plot of the Gene Intensities for All Samples 17

Figure 2.7: Matrix of Gene Expression $\quad 17$

\section{Chapter 4}

Figure 4.1: Index Plot of Genes with Cut-off Points for Existing Outlier Detection

Methods

Figure 4.2: Index Plot of Genes with Cut-off Points for Proposed Outlier Detection

Methods 


\section{List of Tables}

\section{Chapter 4}

Table 4.1: Number of Outliers in Simulation Study

Table 4.2: Number of Outliers in Cancer Data Under Study 


\section{CHAPTER 1}

\section{INTRODUCTION}

\subsection{Outliers in Statistical Data}

Statistical data analysis usually begins with the gathering of observations from a certain population. However, this process of accumulating the data is subject to numerous sources of error. Therefore, the data collected may comprise with some unusually small or large observations, so-called outliers. Determining whether a data set contains one or more outliers is a challenge commonly faced in applied statistics. This is a mostly difficult mission if the properties of the underlying population are not known. However, in many empirical investigations, the assumption that the data come from a particular population is too restrictive or unrealistic. Although outliers are often considered as an error or noise, they may convey important information. Detected outliers are candidates for peculiar data that may otherwise adversely lead to model misspecification, biased parameter estimation and incorrect results. It is therefore important to identify them prior to modeling and analysis.

Outlier detection methods have been suggested for numerous applications, such as credit card fraud detection, clinical trials, voting irregularity analysis, data cleansing, network intrusion, severe weather prediction, geographic information systems, athlete performance analysis, and other data-mining tasks [Hawkins, 1980; Barnett and Lewis, 1994; Ruts and Rousseeuw, 1996; Fawcett and Provost, 1997; Johnson et al., 1998; Penny and Jolliffe, 2001; Acuna and Rodriguez, 2004; Liu et al., 2004; Hadi, Imon and Werner, 2009]. 


\subsection{Gene Expression Data}

The rapid developments of technologies that generate arrays of gene data enable a global view of the transcription levels of hundreds of thousands of genes simultaneously. The outlier detection problem for gene data has its importance but together with the difficulty of high dimensionality. The scarcity of data in high-dimensional space makes each point a relatively good outlier in the view of traditional distance-based definitions. Thus, finding outliers in high dimensional data is more complex.

Microarray technology is used in a wide variety of settings for detecting differential gene expression. Classic statistical issues such as appropriate test statistics, sample size, replicate structure, statistical significance, and outlier detection enter into the design and analysis of gene expression studies. Adding to the complexity is the fact that the number of samples $I$ in a microarray experiment is inevitably much less than the number of genes $\mathbf{J}$ under investigation and that $\mathrm{J}$ is often on the scale of tens of thousands, thus creating a tremendous multiple testing burden.

\subsection{The Need for Detection of Outliers in Gene Expression Data}

Fundamental to the task of analyzing gene expression data is the need to identify genes whose patterns of expression differ according to phenotype or experimental condition. Gene expression is a well-coordinated system, and hence measurements on different genes are in general not independent. Given more complete knowledge of the specific interactions and transcriptional controls, it is conceivable that meaningful comparisons between samples can be made by considering the joint distribution of specific sets of genes. However, the high dimension of gene expression space prohibits a comprehensive exploration, while the fact that our 
understanding of biological systems is only in its infancy means that in many cases we do not know which relationships are important and should be studied. In current practice, differential expression analysis will therefore at least start with a gene-by-gene approach, ignoring the dependencies between genes. A simple approach is to select genes using a fold-change criterion. This may be the only possibility in cases where no, or very few replicates, are available. An analysis solely based on fold change however does not allow the assessment of significance of expression differences in the presence of biological and experimental variation, which may differ from gene to gene. This is the main reason for using statistical tests to assess differential expression. Generally, one might look at various properties of the distributions of a gene's expression levels under different conditions, though most often location parameters of these distributions, such as the mean is considered. Parametric test, such as the t-test, is commonly used. Parametric tests usually have a higher power if the underlying model assumptions, such as normality in the case of the test, are at least approximately fulfilled. Presence of outliers may often destroy normality pattern so it is essential to identify outliers in gene expression data before any further statistical analysis.

\subsection{Outline of the Study}

We organize this thesis in the following way. In chapter 2, we introduce different concepts regarding gene expression. We also introduce the real data which we analyze later for outlier detection. In chapter 3 we introduce the concept of outliers, its consequences. We also introduce some commonly used outlier detection techniques here. In chapter 4, we consider outlier detection methods which are suggested exclusively for gene expression data. The most widely used method for detecting differential gene expression in comparative microarray studies 
is the two-sample t-statistic. A gene is determined to be significant if the absolute t-value exceeds a certain threshold $\mathrm{c}$, which is usually determined by its corresponding P-value or false discovery rate. Recently, Tomlins et al (2005) introduced the cancer outlier profile analysis (COPA) method for detecting cancer genes which are differentially expressed in a subset of disease samples. Heterogeneous patterns of oncogene activation were observed in the majority of cancer types considered in their studies. Thereafter, several further studies in this direction have been proposed. Tibshirani and Hastie (2007) introduced the outlier sums (OS) method, Wu (2007) proposed the outlier robust t-statistic (ORT), Lian (2008) introduced the maximum ordered subset t-statistics (MOST) and a simple statistical test named least sum of ordered subset square t-statistic (LSOSS) is proposed by Wang and Rekaya (2010) for detecting cancer outlier differential gene expression. In this chapter we investigate the performance of the existing methods and introduced three new methods. The effectiveness of our proposed method together with the existing methods is then investigated through a Monte Carlo simulation and also for tumor colon cancer data. 


\section{CHAPTER 2 \\ GENE EXPRESSION AND ITS PATTERN}

In this chapter we briefly discuss gene expression and its pattern and introduce the cancer data that we use in our study. Information regarding gene expression and its pattern is taken from Wikipedia http://en.wikipedia.org/wiki/Gene_expression.

\subsection{Genes}

Genes are subunits of DNA, the information database of a cell that is contained inside the cell nucleus. This DNA carries the genetic blueprint that is used to make all the proteins the cell needs. Every gene contains a particular set of instructions that code for a specific protein. DNA exists in the form of two long, paired strands that form a double helix. Each of these strands is made up of individual building blocks called nucleotide bases. These bases include adenine, thymine, cytosine, and guanine (A, T, C and G), which are arranged in triplets, with each triplet representing a specific amino acid.

DNA is found in all cells present in the body aside from those that do not contain a nucleus such as mature red blood cells or the cornified cells of nails and skin. Each human cell is made up of 46 chromosomes, each of which contains highly condensed and coiled DNA consisting of millions of gene sequences. In each cell, 23 chromosomes are inherited from the father and 23 are inherited from the mother. Twenty-two of the chromosomes from each parent are autosomes and the remaining chromosome is an $X$ or $Y$ sex chromosome. 


\subsection{Gene Expression}

The phenotypic manifestation of a gene or genes by the processes of genetic transcription and genetic translation. Gene expression is the process by which genetic instructions are used to synthesize gene products. These products are usually proteins, which go on to perform essential functions as enzymes, hormones and receptors, for example. Genes that do not code for proteins such as ribosomal RNA or transfer RNA code for functional RNA products. Gene expression analysis is the determination of the pattern of genes expressed at the level of genetic transcription, under specific circumstances or in a specific cell.

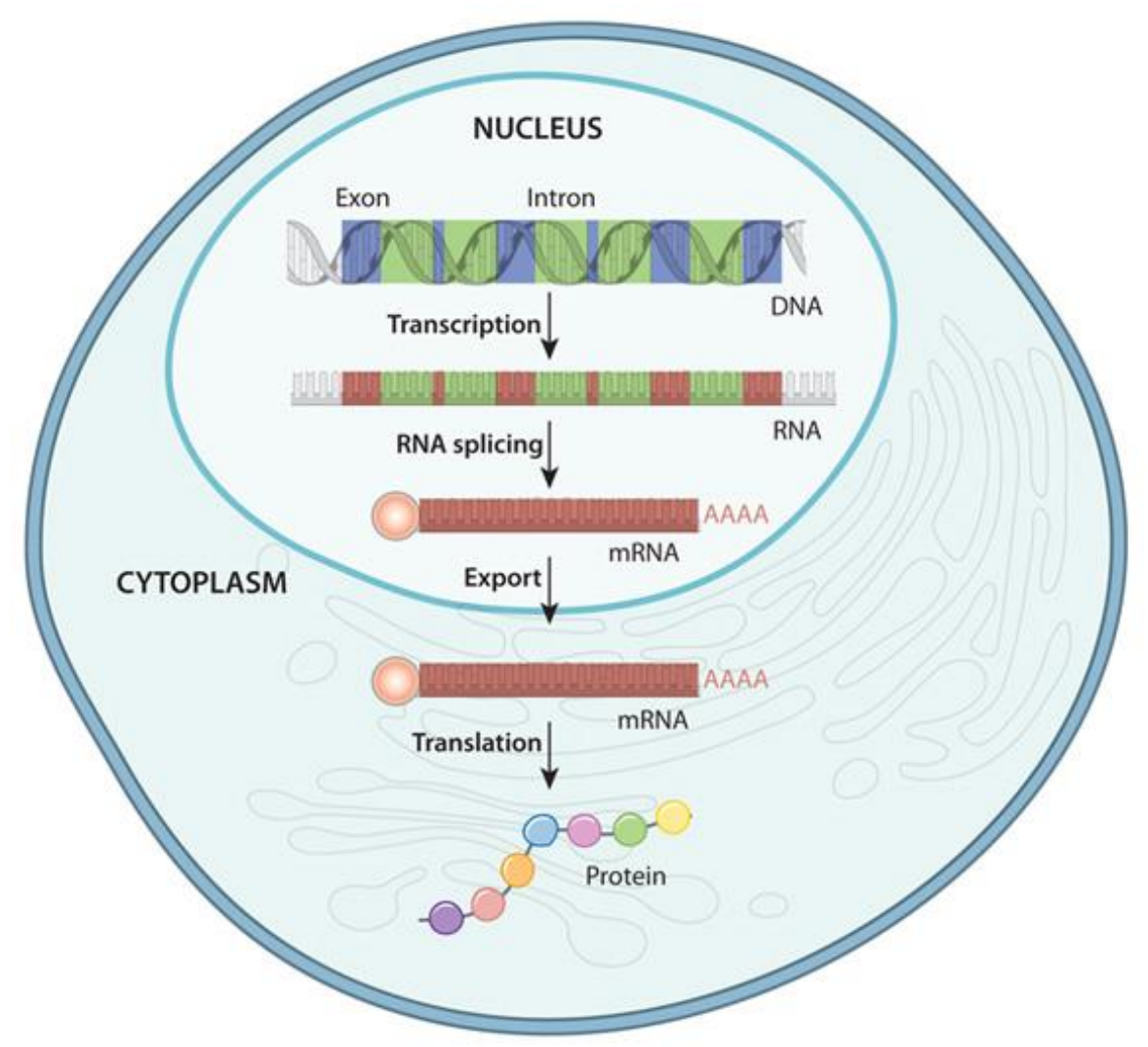

Figure 2.1: An overview of the flow of information from DNA to protein 
When genes are expressed, the genetic information (base sequence) on DNA is first copied to a molecule of mRNA (transcription). The mRNA molecules then leave the cell nucleus and enter the cytoplasm, where they participate in protein synthesis by specifying the particular amino acids that make up individual proteins (translation).

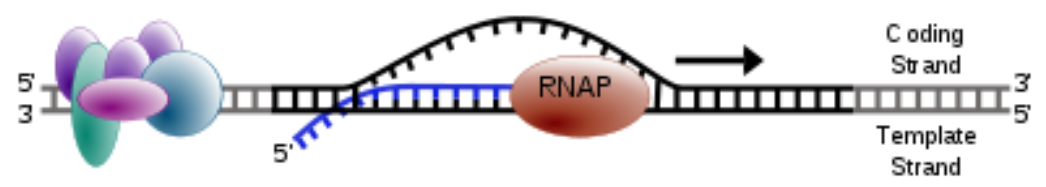

Figure 2.2: The RNA sequence

The process of transcription is carried out by RNA polymerase (RNAP), which uses DNA (black) as a template and produces RNA (blue).

\subsection{Transcription}

A gene is a stretch of DNA that encodes information. Genomic DNA consists of two antiparallel and reverse complementary strands, each having 5' and 3 ' ends. With respect to a gene, the two strands may be labeled the "template strand," which serves as a blueprint for the production of an RNA transcript, and the "coding strand," which includes the DNA version of the transcript sequence. The production of RNA copies of the DNA is called transcription, and is performed in the nucleus by RNA polymerase, which adds one RNA nucleotideat a time to a growing RNA strand. This RNA is complementary to the template $3^{\prime} \rightarrow 5^{\prime}$ DNA strand,[1] which is itself complementary to the coding $5^{\prime} \rightarrow 3^{\prime}$ DNA strand. Therefore, the resulting $5^{\prime} \rightarrow 3^{\prime}$ RNA strand is identical to the coding DNA strand with the exception that thymines (T) are replaced 
with uracils (U) in the RNA. A coding DNA strand reading "ATG" is indirectly transcribed through the non-coding strand as "AUG" in RNA.

Transcription in prokaryotes is carried out by a single type of RNA polymerase, which needs a DNA sequence called a Pribnow box as well as a sigma factor ( $\sigma$ factor) to start transcription. In eukaryotes, transcription is performed by three types of RNA polymerases, each of which needs a special DNA sequence called the promoter and a set of DNA-binding proteins - transcription factors - to initiate the process. RNA polymerase I is responsible for transcription of ribosomal RNA (rRNA) genes. RNA polymerase II (Pol II) transcribes all protein-coding genes but also some non-coding RNAs (e.g., snRNAs, snoRNAs or long non-coding RNAs). Pol II includes a C-terminal domain (CTD) that is rich in serine residues. When these residues are phosphorylated, the CTD binds to various protein factors that promote transcript maturation and modification. RNA polymerase IIItranscribes 5S rRNA, transfer RNA (tRNA) genes, and some small non-coding RNAs (e.g., 7SK). Transcription ends when the polymerase encounters a sequence called theterminator.

While transcription of prokaryotic protein-coding genes creates messenger RNA (mRNA) that is ready for translation into protein, transcription of eukaryotic genes leaves aprimary transcript of RNA (pre-mRNA), which first has to undergo a series of modifications to become a mature mRNA. These include 5' capping, which is set of enzymatic reactions that add 7-methylguanosine $(\mathrm{m} 7 \mathrm{G})$ to the 5 ' end of pre-mRNA and thus protect the RNA from degradation byexonucleases. The $\mathrm{m} 7 \mathrm{G}$ cap is then bound by cap binding complex heterodimer (CBC20/CBC80), which aids in mRNA export to cytoplasm and also protect the RNA from decapping. 
Another modification is 3 ' cleavage and polyadenylation. They occur if polyadenylation signal sequence (5'- AAUAAA-3') is present in pre-mRNA, which is usually between proteincoding sequence and terminator. The pre-mRNA is first cleaved and then a series of $\sim 200$ adenines (A) are added to form poly(A) tail, which protects the RNA from degradation. Poly(A) tail is bound by multiple poly(A)-binding proteins (PABP) necessary for mRNA export and translation re-initiation.

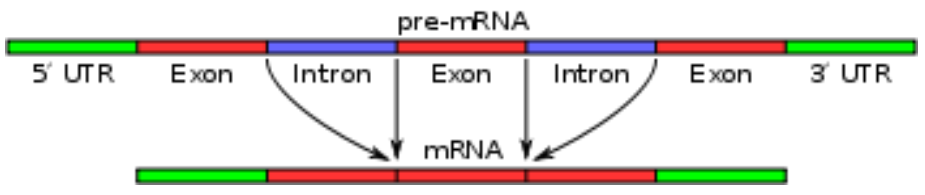

Figure 2.3: Simple illustration of exons and introns in pre-mRNA and the formation of mature mRNA by splicing

A very important modification of eukaryotic pre-mRNA is RNA splicing. The majority of eukaryotic pre-mRNAs consist of alternating segments called exons and introns. During the process of splicing, an RNA-protein catalytical complex known as spliceosome catalyzes two transesterification reactions, which remove an intron and release it in form of lariat structure, and then splice neighbouring exons together. In certain cases, some introns or exons can be either removed or retained in mature mRNA. This so-called alternative splicing creates series of different transcripts originating from a single gene. Because these transcripts can be potentially translated into different proteins, splicing extends the complexity of eukaryotic gene expression. Extensive RNA processing may be an evolutionary advantage made possible by the nucleus of eukaryotes. In prokaryotes transcription and translation happen together whilst in eukaryotes the nuclear membrane separates the two processes giving time for RNA processing to occur. 


\subsection{Non-coding RNA Maturation}

In most organisms non-coding genes (ncRNA) are transcribed as precursors that undergo further processing. In the case of ribosomal RNAs (rRNA), they are often transcribed as a prerRNA that contains one or more rRNAs. The pre-rRNA is cleaved and modified (2'-Omethylation and pseudouridine formation) at specific sites by approximately 150 different small nucleolus-restricted RNA species, called snoRNAs. SnoRNAs associate with proteins, forming snoRNPs. While snoRNA part basepair with the target RNA and thus position the modification at a precise site, the protein part performs the catalytical reaction. In eukaryotes, in particular a snoRNP called RNase, MRP cleaves the 45S pre-rRNA into the 28S, 5.8S, and $18 \mathrm{~S}$ rRNAs. The rRNA and RNA processing factors form large aggregates called the nucleolus.

In the case of transfer RNA (tRNA), for example, the 5' sequence is removed by RNase $\mathrm{P}$, whereas the $3^{\prime}$ end is removed by the tRNase $\mathrm{Z}$ enzyme and the non-templated 3' CCA tail is added by a nucleotidyl transferase. In the case of micro RNA (miRNA), miRNAs are first transcribed as primary transcripts or pri-miRNA with a cap and poly-A tail and processed to short, 70-nucleotide stem-loop structures known as pre-miRNA in the cell nucleus by the enzymes Drosha and Pasha. After being exported, it is then processed to mature miRNAs in the cytoplasm by interaction with the endonuclease Dicer, which also initiates the formation of the RNA-induced silencing complex (RISC), composed of theArgonaute protein.

Even snRNAs and snoRNAs themselves undergo series of modification before they become part of functional RNP complex. This is done either in the nucleoplasm or in the specialized compartments called Cajal bodies. Their bases are methylated or pseudouridinilated by a group of small Cajal body-specific RNAs (scaRNAs), which are structurally similar to snoRNAs. 
In eukaryotes most mature RNA must be exported to the cytoplasm from the nucleus. While some RNAs function in the nucleus, many RNAs are transported through the nuclear pores and into the cytosol. Notably this includes all RNA types involved in protein synthesis. In some cases RNAs are additionally transported to a specific part of the cytoplasm, such as a synapse; they are then towed by motor proteins that bind through linker proteins to specific sequences (called "zipcodes") on the RNA.

\subsection{Translation}

During the translation, tRNA charged with amino acid enters the ribosome and aligns with the correct mRNA triplet. Ribosome then adds amino acid to growing protein chain.

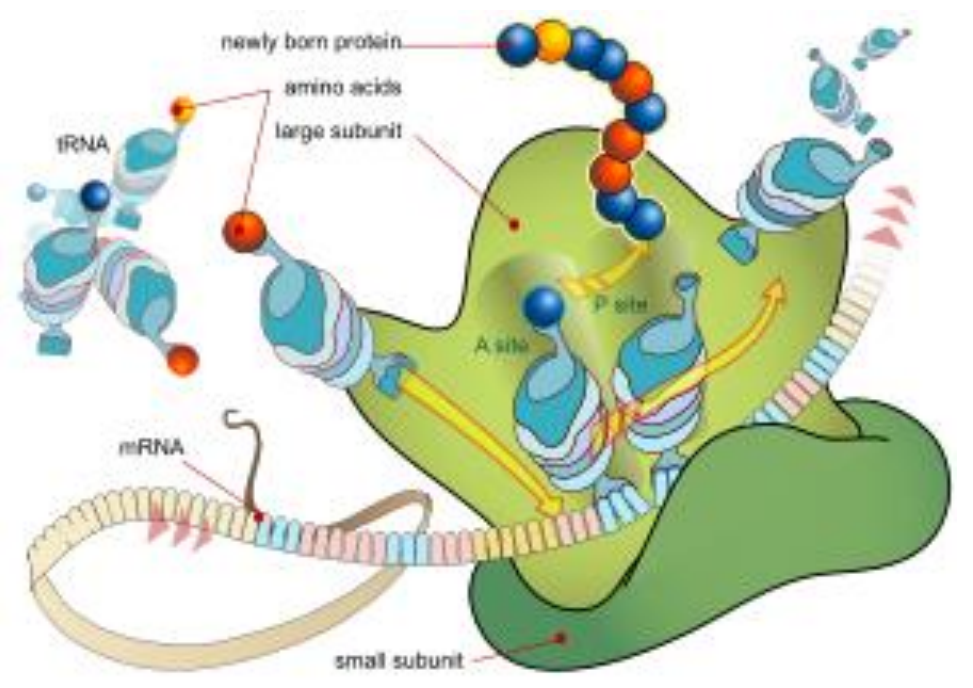

Figure 2.4: Protein chain

For some RNA (non-coding RNA) the mature RNA is the final gene product. In the case of messenger RNA (mRNA) the RNA is an information carrier coding for the synthesis of one or more proteins. mRNA carrying a single protein sequence (common in eukaryotes) 
is monocistronic whilst mRNA carrying multiple protein sequences (common in prokaryotes) is known as polycistronic.

Every mRNA consists of three parts - 5' untranslated region (5'UTR), protein-coding region or open reading frame (ORF) and 3' untranslated region (3'UTR). The coding region carries information for protein synthesis encoded by the genetic code to form triplets. Each triplet of nucleotides of the coding region is called a codon and corresponds to a binding site complementary to an anticodon triplet in transfer RNA. Transfer RNAs with the same anticodon sequence always carry an identical type of amino acid. Amino acids are then chained together by the ribosome according to the order of triplets in the coding region. The ribosome helps transfer RNA to bind to messenger RNA and takes the amino acid from each transfer RNA and makes a structure-less protein out of it. Each mRNA molecule is translated into many protein molecules, on average $\sim 2800$ in mammals.

In prokaryotes translation generally occurs at the point of transcription (cotranscriptionally), often using a messenger RNA that is still in the process of being created. In eukaryotes translation can occur in a variety of regions of the cell depending on where the protein being written is supposed to be. Major locations are the cytoplasm for soluble cytoplasmic proteins and the membrane of the endoplasmic reticulum for proteins that are for export from the cell or insertion into a cell membrane. Proteins supposed to express at the endoplasmic reticulum are recognised part-way through the translation process. This is governed by the signal recognition particle - a protein that binds to the ribosome and directs it to the endoplasmic reticulum when it finds a signal peptide on the growing (nascent) amino acid chain. Translation is the communication of the meaning of a source-language text by means of an equivalent target-language text. 


\subsection{Folding}

The polypeptide folds into its characteristic and functional three-dimensional structure from a random coil. Each proteinexists as an unfolded polypeptide or random coil when translated from a sequence of mRNA into a linear chain of amino acids. This polypeptide lacks any developed three-dimensional structure (the left hand side of the neighboring figure). Amino acids interact with each other to produce a well-defined three-dimensional structure, the folded protein (the right hand side of the figure) known as the native state. The resulting threedimensional structure is determined by the amino acid sequence (Anfinsen's dogma).

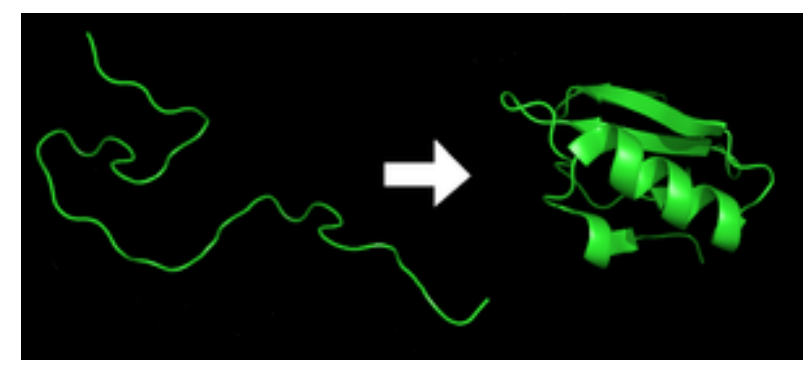

Figure 2.5: Protein before (left) and after (right) folding.

The correct three-dimensional structure is essential to function, although some parts of functional proteins may remain unfolded Failure to fold into the intended shape usually produces inactive proteins with different properties including toxicprions. Several neurodegenerative and other diseases are believed to result from the accumulation of misfolded (incorrectly folded) proteins. Many allergies are caused by the folding of the proteins, for the immune system does not produce antibodies for certain protein structures.

Enzymes called chaperones assist the newly formed protein to attain (fold into) the 3dimensional structure it needs to function. Similarly, RNA chaperones help RNAs attain their 
functional shapes. Assisting protein folding is one of the main roles of the endoplasmic reticulum in eukaryotes.

\subsection{Translocation}

Secretory proteins of eukaryotes or prokaryotes must be translocated to enter the secretory pathway. Newly synthesized proteins are directed to the eukaryotic Sec61 or prokaryotic SecYEG translocation channel by signal peptides. Whereas the efficiency of protein secretion in eukaryotes is very dependent on the signal peptide which has been used.

Many proteins are destined for other parts of the cell than the cytosol and a wide range of signalling sequences or (signal peptides) are used to direct proteins to where they are supposed to be. In prokaryotes this is normally a simple process due to limited compartmentalisation of the cell. However in eukaryotes there is a great variety of different targeting processes to ensure the protein arrives at the correct organelle. Not all proteins remain within the cell and many are exported, for example digestive enzymes, hormones and extracellular matrix proteins. In eukaryotes the export pathway is well developed and the main mechanism for the export of these proteins is translocation to the endoplasmic reticulum, followed by transport via the Golgi apparatus.

\subsection{Cancer Data Under Study}

For this study we use the data pertaining to the article "Broad patterns of gene expression revealed by clustering of tumor and normal colon tissues probed by oligonucleotide arrays" by Alon et al. (1999). The matrix http://genomics-pubs.princeton.edu/oncology/affydata/I2000.html contains the expression of the 2000 genes with highest minimal intensity across the 62 tissues. 
The genes are placed in order of descending minimal intensity. Each entry in I2000 is a gene intensity derived from the $\sim 20$ feature pairs that correspond to the gene on the chip, derived using the filtering process. The data is otherwise unprocessed (for example it has not been normalized by the mean intensity of each experiment).

The file http://genomics-pubs.princeton.edu/oncology/affydata/names.html contains the EST number and description of each of the 2000 genes, in an order that corresponds to the order in I2000. Note that some ESTs are repeated which means that they are tiled a number of times on the chip, with different choices of feature sequences. The descriptions UMGAP, HSAC07 and I correspond to control RNAs spiked with each experiment. The identity of the 62 tissues is given in file http://genomics-pubs.princeton.edu/oncology/affydata/tissues.html. The numbers correspond to patients, a positive sign to a normal tissue, and a negative sign to a tumor tissue. There were 22 tissues from normal sample 40 tissues from tumor sample.

\subsection{Patterns of Gene Expression of Tumor and Normal Colon Tissues}

Gene expression in 40 tumor and 22 normal colon tissue samples was taken from Gene expression in 40 tumor and 22 normal colon tissue samples was analyzed with an Affymetrix oligonucleotide array complementary to more than 6,500 human genes. An efficient two-way clustering algorithm was applied to both the genes and the tissues, revealing broad coherent patterns that suggest a high degree of organization underlying gene expression in these tissues. Coregulated families of genes clustered together, as demonstrated for the ribosomal proteins. Clustering also separated cancerous from noncancerous tissue and cell lines from in vivo tissues on the basis of subtle distributed patterns of genes even when expression of individual genes varied only slightly between the tissues. For the study the 2,000 genes with highest minimal 
intensity across the tissues were used. To get an idea about the gene expression in normal sample and tumor sample we construct an index plot of the genes for normal, tumor and all samples which is presented in Figure 2.6. It seems to us from this figure that tumor samples have higher intensities than normal samples. The ranges of normal genes are 5.82 to 14173.05 whereas for tumor samples these values are 5.89 to 20903.18 .

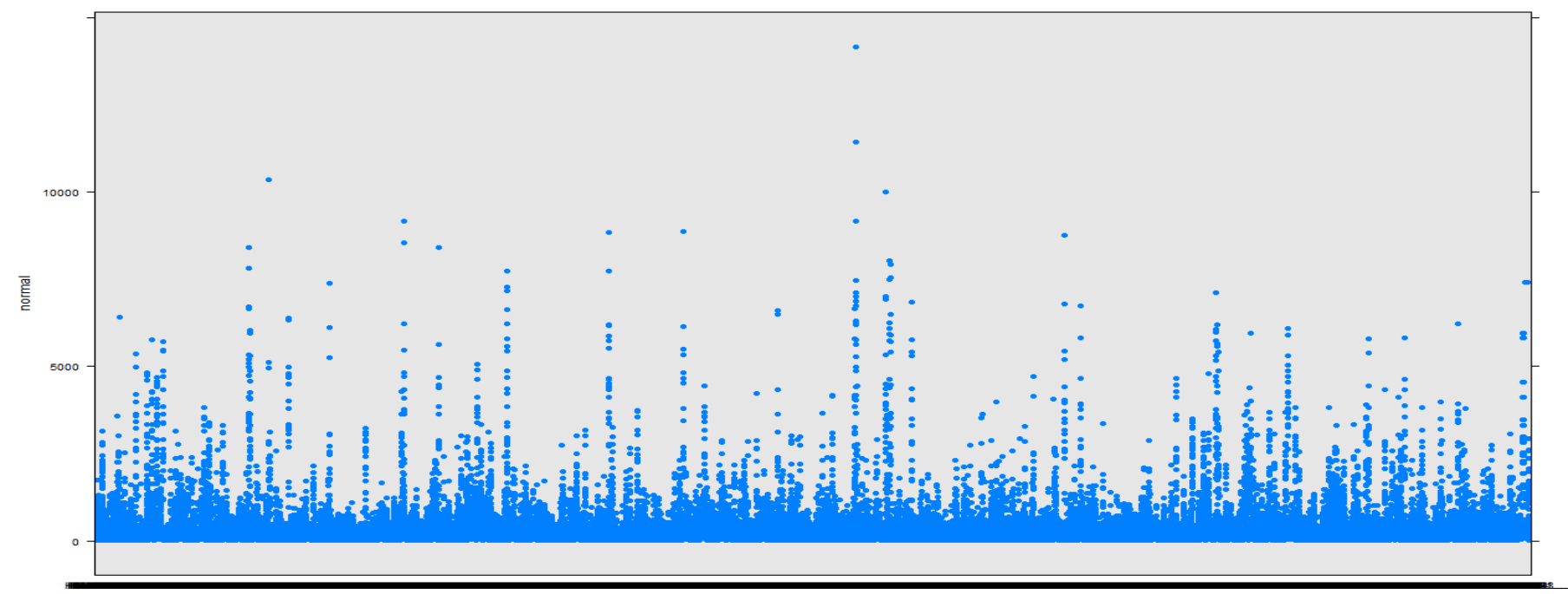

Figure 2.6 (a): Plot of the Gene Intensities for Normal Samples (Horizontal-gene index, vertical intensities)

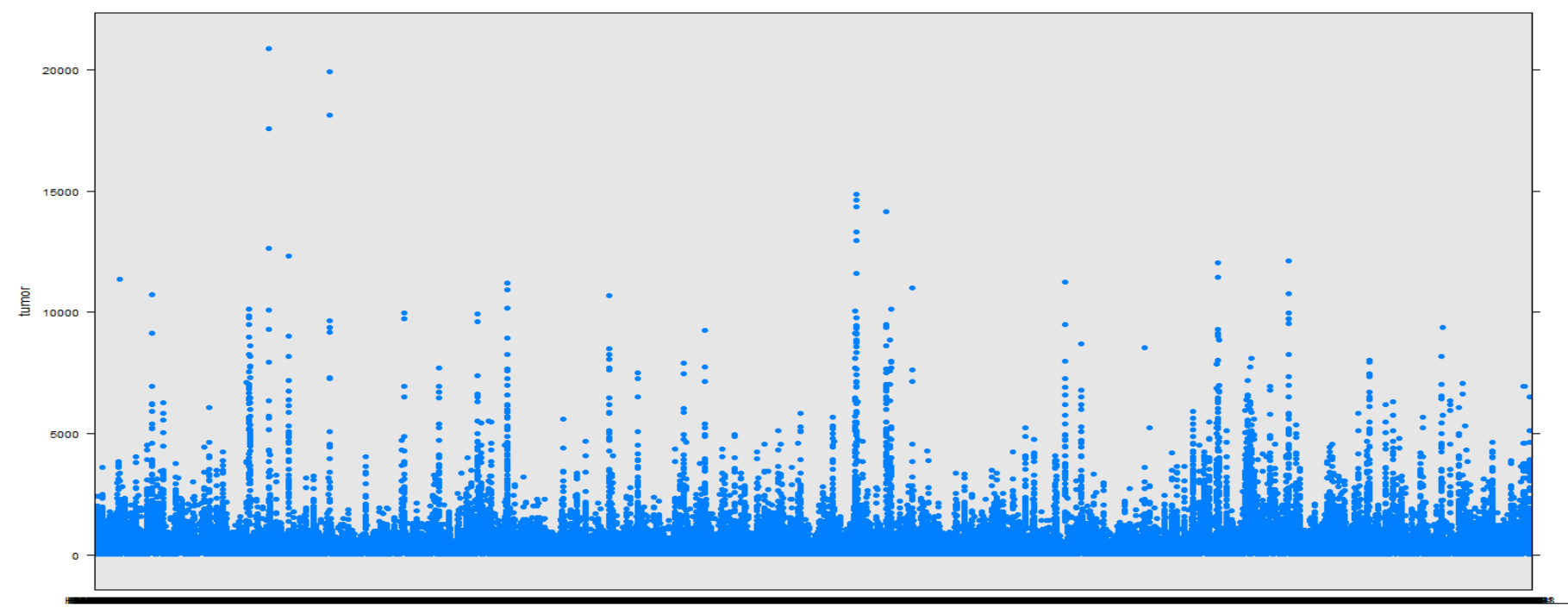

Figure 2.6 (b): Plot of the Gene Intensities for Tumor Samples (Horizontal-gene index, vertical intensities) 


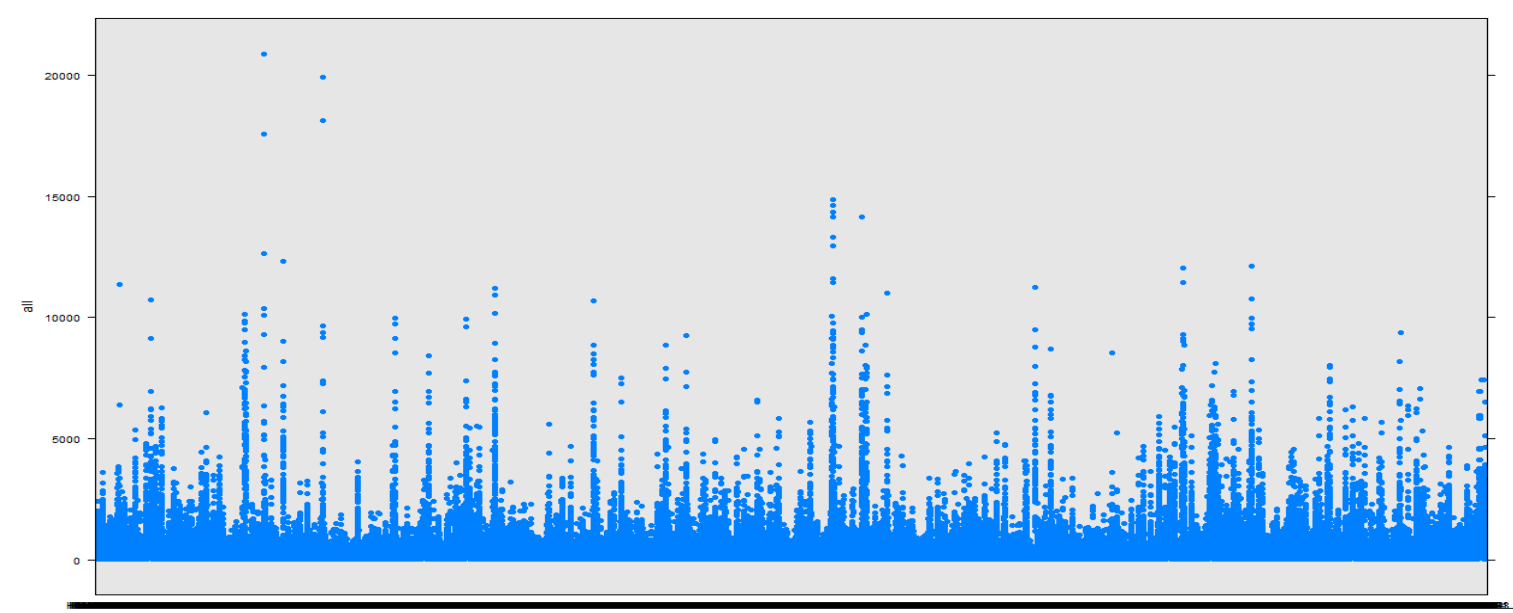

Figure 2.6 (c): Plot of the Gene Intensities for All Samples (Horizontal-gene index, vertical intensities)

The index plots of the genes according to different group indicate that the intensity level in the tumor samples usually much higher than that of normal samples. This indication is clearly a good sign to doubt that there must be some unusual pattern of heterogeneity in the tumor samples. Our attempt to detect outlier has got a strong background to proceed on.

A Unclustered

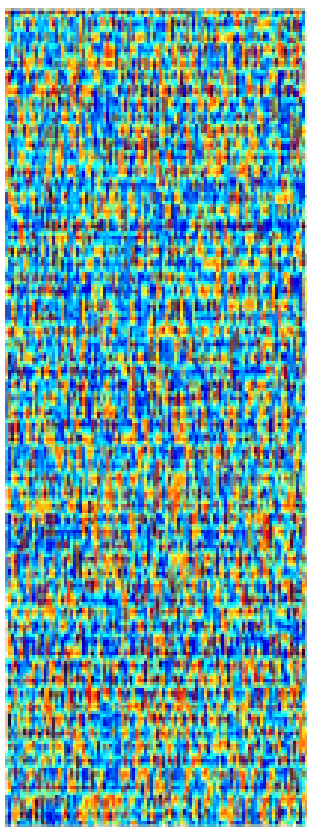

B Clustered

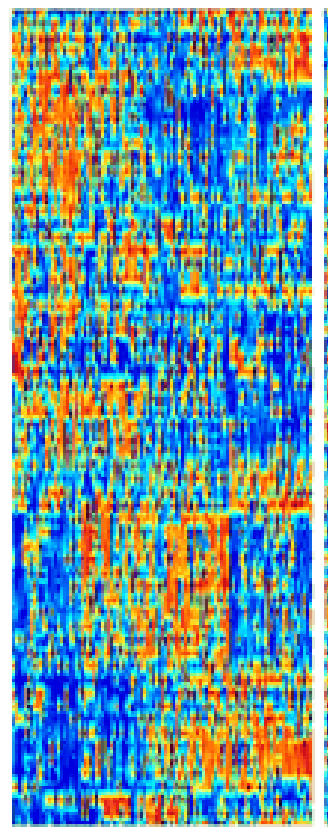

C Random Unclustered Random Clustered

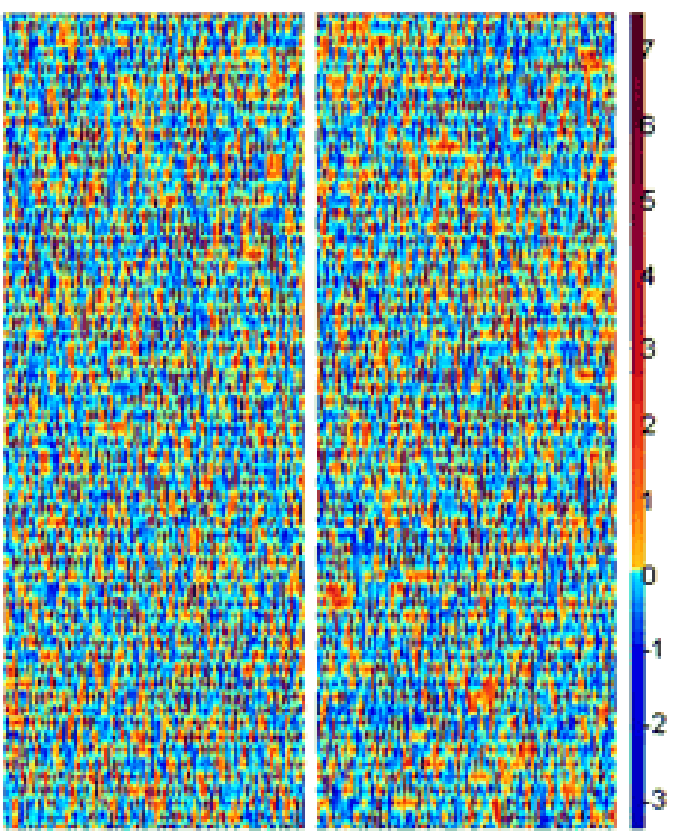

Figure 2.7: matrix of gene expression 
We have taken Figure 2.7 from "Broad patterns of gene expression revealed by clustering analysis of tumor and normal colon tissues probed by oligonucleotide arrays" by A Ulon, a proceedings of the National Academy of Sciences of the United States of America. The vertical axis corresponds to genes, and the horizontal axis to tissues. Each gene was normalized so its average intensity across the tissues is 0 , and its SD is 1 . The color code used is indicated in the adjoining scale. (A) Unclustered data set. (B) Clustered data. The 62 tissues are arranged on the vertical axis according to the ordered tree of Fig. 3. The 2,000 genes are arranged on the horizontal axis according to their ordered tree. $(C)$ Unclustered randomized data, where the original data set was randomized (the location of each number in the matrix was randomly shifted). (D) Clustered randomized data, subjected to the same clustering algorithm as in B.

The 2,000 genes are arranged on the horizontal axis according to their ordered tree. We will use this data again in Chapter 4 for finding outliers in gene expression. 


\section{CHAPTER 3 \\ IDENTIFICATON OF OUTLIERS}

Given a data set, outlier detection aims at finding data points which are very different from the remainder. The concept of outlier came from Astrophysics even before the formal development of statistics and statistical techniques.

\subsection{Outliers}

The term 'outlier' was used in astrophysics to distinguish planets which are 'outlying' in our solar system. This field has received a large attention in the last decades because outliers often represent critical information about an abnormal behavior of the system described by the data. Outliers are also called: event, novelty, anomaly, noise, deviation or exception. However there is no formal definition of an outlier because this intuitive notion varies with the context and the desired characteristics of outliers. In a statistical perspective, Grubbs (1969) defined that "an outlying observation, or outlier, is one that deviates markedly from other members of the sample in which it occurs". Hawkins (1980) defines an outlier as "an observation which deviates so much from other observations as to arouse suspicions that it was generated by a different mechanism", while Barnett and Lewis (1994) call an outlier "an observation (or subset of observations) which appears to be inconsistent with the remainder of that set of data". They also mentioned 'Even before the formal development of statistical method, argument raged over whether, and on what basis, we should discard observations from a set of data on the grounds that they are 'unrepresentative', 'spurious', or 'mavericks' or 'rogues'." 
Outliers do not inevitably 'perplex' or 'mislead'; they are not necessarily 'bad' or 'erroneous', and the experimenter may be tempted in some situations not to reject an outlier but to welcome it as an indication of some unexpectedly useful industrial treatment or surprisingly successful agricultural variety. Sometimes it is a matter of subjective judgment on the part of the observer whether or not some observations are genuine members of the main population. If they are contaminants (arising from some other distribution), they may frustrate attempts to draw inferences about the original population. Of course, any contaminants which occur in the midst of the data set will not be conspicuous. We call contaminants to be outliers when they appear surprisingly extreme. Outliers may or may not be contaminants; contaminants may or may not be outliers.

Hampel et al. (1986) claim that a routine data set typically contains about 1-10\% outliers, and even the highest quality data set cannot be guaranteed free of outliers. One immediate consequence of the presence of outliers is that they may cause apparent non-Normality and the entire classical inferential procedure might breakdown in the presence of outliers.

There are mainly three different sources of outliers

- Inherent Variability: Natural feature of a population that is uncontrollable.

- Measurement Error: The rounding of obtained values or mistakes in recording compound measurement error.

- Execution Error: Imperfect collection of data. We may inadvertently choose a biased sample or include individuals not truly representative of the population we aimed to sample. 


\subsection{Detection of Outliers}

Observations arising from large variation of the inherent type are called outliers, while observations subjected to large measurement error or execution errors are termed spurious observations [Anscombe (1960)]. When we order the sample, the smallest and the largest ordered observations are known as extremes. Whether we declare either of them to be an outlier depends on consideration of how they appear in relation to the postulated model. Extreme values may or may not be outliers. To quote Barnett and Lewis (1994) 'Any outliers, however, are always extreme values in the sample.'

There is no generic approach to detect outliers. Many approaches have been proposed to detect or identify the outliers based on density based, distance based, distribution based and clustering based approaches. In the Density based approach they compute the data with density of regions in which low density regions are identified as outliers. Breunig (2000) proposed the local outlier factor (LOF) as an outlier score for any given data point based on the distance from its local neighborhood. In the distance based approach (Knorr 2000; Angiulli 2005) the outliers are detected by a distance measure on the feature space. Outliers are identified by using k-nearest neighbor method to rank the outliers (Ramasamy, 2000). The problem with this approach is that it is very difficult to find a particular value in a dataset. Distribution based approach (Rosseeuw 1996) had developed statistical methods from the given data and applied statistical test to find the object belong to a particular model or not. Objects with low probabilities are identified as outliers in the statistical model. Because the distribution based approaches are univariate in nature they cannot be applied in multidimensional data space Clustering based approach (Achuna and Rodriguez 2004) identified outliers as clusters of small sizes. The advantage of this approach is that it may not be supervised. Hierarchical based approach (Loureiro, 2004 and Almeida 2006) 
was used to identify the outliers by using the resultant clusters as an indicator to identify the outliers.

\subsubsection{The 'three - sigma' Rule}

If we assume a normal distribution, a single value may be considered as an outlier if it falls outside a certain range of the standard deviation. A traditional measure of the 'outlyingness' of an observation with respect to a sample is the ratio between its distance to the sample mean and the sample SD:

$$
t_{i}=\frac{x_{i}-\bar{x}}{s}, \quad i=1,2, \ldots, n
$$

Observations with $\left|t_{i}\right|>3$ are traditionally deemed as suspicious (the three-sigma rule), based on the fact that they would be very unlikely under normality, since $P(|t|>3)=0.003$ for a random variable $t$ with a standard normal distribution.

\subsubsection{Grubbs' Test}

Grubbs (1969) proposed a test to detect outliers in a univariate data set. It is based on the assumption of normality. Grubbs' test is also known as the maximum normed residual test. The Grubbs test statistic is the largest absolute deviation from the sample mean in units of the sample standard deviation. Grubb's Test is a test based on normal distribution, the effects of which are that the data analyzed with this method should have normal distribution. The name of this test comes from the author's name -Frank Grubbs. This test should be performed as long as all outliers will be detected. In this test we have two hypotheses: the null-hypothesis $\left(H_{0}\right)$ and the alternative hypothesis $\left(H_{1}\right)$ :

$H_{0}$ : There are no outliers in the data set.

$H_{1}$ : There is at least one outlier in the data set. 
The general formula for Grubb's Test can be presented as follows:

$$
G=\frac{\operatorname{Max}\left|x_{i}-\bar{x}\right|}{s}
$$

The calculated value of $G$ is compared with the critical value for Grubb's Test. For the two-sided test, the hypothesis of no outliers is rejected if

$$
G>\frac{n-1}{\sqrt{n}} \sqrt{\frac{t^{2}((\alpha / 2 n), n-2)}{n-2+t^{2}((\alpha / 2 n), n-2)}}
$$

with $\alpha$ denoting the critical value of the $t$ distribution with $n-2$ degrees of freedom and a significance level of $\alpha /(2 n)$. When the calculated value is higher or lower than the critical value for the chosen statistical significance, then the calculated value can be accepted as an outlier. I

\subsubsection{Dixon's Q-test}

This method was proposed by Dean and Dixon (1951). This test has some restrictions - it is impossible to use this test with a big data set. The Dixon's Q-test is a very simple test for outliers when we suspect that outliers are extreme observations in the data set. Q-test is based on the statistical distribution of "subrange ratios" of ordered data samples, drawn from the same normal population. Hence, a normal distribution of data is assumed whenever this test is applied. The test is very simple and it is applied as follows:

1. The $n$ values comprising the set of observations under examination are arranged in ascending order: $x_{(1)}<x_{(2)}<\ldots<x_{(n)}$.

2. The statistic $\mathrm{Q}$ is a ratio defined as the difference of the suspect value from its nearest one divided by the range of the values. Thus, for testing $x_{(1)}$ or $x_{(n)}$ (as possible outliers) we use the following values: 


$$
\mathrm{Q}=\frac{x_{(2)}-x_{(1)}}{x_{(n)}-x_{(1)}} \quad \text { or } \quad \frac{x_{(n)}-x_{(n-1)}}{x_{(n)}-x_{(1)}}
$$

3. The obtained Qobs value is compared to a critical Q-value (Qcrit) found in tables.

4. If Qobs > Qcrit, then the suspect value can be characterized as an outlier.

\subsection{Robust Outlier Detection Methods}

In many cases, presence of outliers may make the diagnostic procedure unreliable for which masking and/or swamping can occur. So we need detection methods which are not affected by outliers. The word "robust" literary means something "very strong." So robust statistics are those statistics which do not breakdown easily. The term robustness signifies insensitivity to small deviations from the assumption. That means a robust procedure is nearly as efficient as the classical procedure when classical assumptions hold strictly but is considerably more efficient over all when there is a small departure from them. One objective of robust techniques is to cope with outliers by trying to keep small the effects of their presence. Consequently, we should require resistant estimators (Tukey, 1977). The analogous term used in the literature: resistant statistics.

Here we introduce several statistics which are robust in the presence of outliers. Median and trimmed mean are robust measures of location. For the measure of dispersion we can use the normalized median absolute deviation (MADN). For a set of data the median absolute deviation (MAD) is defined as

$$
\operatorname{MAD}(x)=\operatorname{Med}\{|x-\operatorname{Med}(x)|\}
$$

To make the MAD comparable to the SD in terms of efficiency, we consider the normalized MAD defined as

$$
\operatorname{MADN}(x)=\operatorname{MAD}(x) / 0.6745
$$


Two other well-known dispersion estimates are the range defined as

$$
R=x_{(n)}-x_{(1)}
$$

and the inter-quartile range (IQR) defined as

$$
\operatorname{IQR}(x)=Q_{3}-Q_{1}
$$

Both of them are based on order statistics; (3.7) is clearly very sensitive to outliers, while (3.8) is not.

\subsubsection{Robust t like Statistic}

Let us now use the robust plug-in technique Imon, Midi and Rana (2013) to obtain a robust t-like statistic by replacing mean by median and SD by the normalized median absolute deviation (MADN). Thus the modified statistic becomes

$$
t_{i}^{\prime}=\frac{x_{i}-\operatorname{Median}(x)}{\operatorname{MADN}(x)}
$$

Observations with $\left|t_{i}^{\prime}\right|>3$ are identified as outliers.

\subsubsection{Test Based on the Interquartile Range}

The above-mentioned strategies for identifying outliers are probably most appropriate for symmetric unimodal distributions. If a distribution is skewed, it is recommended to calculate the threshold for outliers from the interquartile distance:

$$
Q_{1}-1.5 \mathrm{IQR}<x_{i}<Q_{3}+1.5 \mathrm{IQR}
$$

\subsubsection{Hampel's Test}

In recent years Hampel (1984)'s test for outliers has become very popular in data mining and knowledge discovery. According to this rule an observation $x_{i}$ is identified as an outlier if

$$
x_{i}-\operatorname{median}(x)>4.5 \operatorname{MAD}(x)
$$


It is interesting to note that Hampel's test is equivalent to robust $t$ test. Recall that according to the robust $t$ test an observation is identified as an outlier according to (3.9) which yields

$$
x_{i}-\operatorname{median}(\mathrm{x})>3 \operatorname{MADN}(x)=4.4474 \operatorname{MAD}(x)
$$

\subsection{Masking and Swamping Effects}

We often observe that identification methods fail to identify potential outliers or the methods identify cases as outliers which are actually not.

In masking it is said that one outlier masks a second outlier, if the second outlier can be considered as an outlier only by itself, but not in the presence of the first outlier. Thus, after the deletion of the first outlier the second instance is emerged as an outlier. Masking occurs when a cluster of outlying observations skews the mean and the covariance estimates toward it, and the resulting distance of the outlying point from the mean is small.

In describing the swamping effect it is said that one outlier swamps a second observation, if the latter can be considered as an outlier only under the presence of the first one. In other words, after the deletion of the first outlier the second observation becomes a non-outlying observation. Swamping occurs when a group of outlying instances skews the mean and the covariance estimates toward it and away from other non-outlying instances, and the resulting distance from these instances to the mean is large, making them look like outliers. 


\section{CHAPTER 4 \\ IDENTIFICATION OF OUTLIERS IN GENE EXPRESSION \\ DATA}

A gene expression measurement which differs surprisingly from the other measurements obtained for the same gene on other samples of the same class. The outlying principle assumes that the data, with the possible exception of any outlier, form a sample of a given distribution |here the normal distribution. We will use a reasonable test statistical to decide whether or not the suspect measurement is an outlier.

\subsection{Existing Outlier Detection Methods for Gene Expression Data}

Assuming case-control microarray data were generated for detecting differentially expressed genes consisting of $n$ samples from a normal group and $\mathrm{m}$ samples from a cancer group. Let $x_{i j}$ be the expression value for gene $i=(1,2, \ldots, p)$ and sample $j=(1,2, \ldots, n)$ in the normal group and $y_{i j}$ be the expression value for gene $i=(1,2, \ldots, p)$ and sample $j=(1,2, \ldots$, $m$ ) in the cancer group. In this study, and without loss of generality, we are only interested in one-sided tests where the activated genes from cancer samples are over-expressed or upregulated.

\subsection{1 t-statistic}

The two-condition $t$-statistic for gene $i$ is defined by:

$$
t_{i}=\frac{\overline{y_{l}}-\overline{x_{l}}}{s_{i}}
$$

where $\overline{y_{l}}$ is the mean expression value in cancer samples, $\overline{x_{l}}$ is the mean expression value in normal samples for gene $i$ and $s_{i}$ is the pooled standard error estimate given by: 


$$
S_{i}^{2}=\frac{\sum_{1 \leq j \leq n}\left(x_{i j}-\bar{x}_{l}\right)^{2}-\sum_{1 \leq j \leq m}\left(y_{i j}-\bar{y}_{l}\right)^{2}}{n+m-2}
$$

The $t$-statistic is powerful when most cancer samples are activated.

\subsubsection{Cancer Outliers Profile Analysis (COPA) statistic}

Tomlins et al. (2005) defines the COPA statistic as

$$
\operatorname{copa}_{i}=\frac{q_{r}\left(\left\{y_{i j}: 1 \leq j \leq m\right\}\right)-\operatorname{med}_{i}}{\operatorname{mad}_{i}}
$$

where $q_{r}($.$) is the r^{\text {th }}$ percentile of the expression data, and med $_{i}$ is the median expression value for all samples

$$
\operatorname{med}_{i}=\operatorname{median}\left(\left\{\mathrm{x}_{i j}: 1 \leq \mathrm{j} \leq \mathrm{n}\right\},\left\{\mathrm{y}_{i j}: 1 \leq \mathrm{j} \leq \mathrm{m}\right\}\right)
$$

and $\operatorname{mad}_{i}$ is the median absolute deviation of expression values in all samples and is given by:

$$
\operatorname{mad}_{i}=1.4826 \times \operatorname{median}\left(\left\{\left(\mathrm{x}_{i j}-\operatorname{med}_{i}\right): 1 \leq \mathrm{j} \leq \mathrm{n}\right\},\left\{\left(\mathrm{y}_{i j}-\operatorname{med}_{i}\right): 1 \leq \mathrm{j} \leq \mathrm{m}\right\}\right) .
$$

\subsubsection{Outliers Sums (OS) Statistic}

COPA statistic uses a fixed $r^{\text {th }}$ sample percentile, which is determined by users. This limitation was overcome by the OS statistic defined by Tibshirani and Hastie (2007) as:

$$
O S_{i}=\frac{\sum_{y_{i j} \in R_{i}}\left(y_{i j}-\operatorname{med}_{i}\right)}{\operatorname{mad}_{i}}
$$

where

$\mathrm{R}_{i}=\left\{y_{i j}: y_{i j}>\mathrm{q}_{75}\left(\left\{x_{i j}: 1 \leq \mathrm{j} \leq \mathrm{n}\right\},\left\{y_{i j}: 1 \leq \mathrm{j} \leq \mathrm{m}\right\}\right)+\operatorname{IQR}\left(\left\{x_{i j}: 1 \leq \mathrm{j} \leq \mathrm{n}\right\},\left\{y_{i j}: 1 \leq \mathrm{j} \leq\right.\right.\right.$ $\mathrm{m}\})\}(4.5)$

and $\operatorname{IQR}(\bullet)$ is the inter-quantile range of the expression data

$$
\begin{aligned}
\operatorname{IQR}\left(\left\{x_{i j}: 1 \leq \mathrm{j}\right.\right. & \left.\leq \mathrm{n}\},\left\{y_{i j}: 1 \leq \mathrm{j} \leq \mathrm{m}\right\}\right)=\mathrm{q}_{75}\left(\left\{x_{i j}: 1 \leq \mathrm{j} \leq \mathrm{n}\right\},\left\{y_{i j}: 1 \leq \mathrm{j} \leq \mathrm{m}\right\}\right)-\mathrm{q}_{25}\left(\left\{x_{i j}: 1\right.\right. \\
& \left.\leq \mathrm{j} \leq \mathrm{n}\},\left\{y_{i j}: 1 \leq \mathrm{j} \leq \mathrm{m}\right\}\right)
\end{aligned}
$$




\subsubsection{Outliers Robust t (ORT) statistic}

Wu (2007) modified the OS statistic by proposing the ORT statistic which consists mainly in changing the definition of $R_{i}$ as:

$$
\mathrm{R}_{i}=\left\{y_{i j}: y_{i j}>\mathrm{q}_{75}\left(\left\{x_{i j}: 1 \leq \mathrm{j} \leq \mathrm{n}\right\}\right)+\operatorname{IQR}\left(\left\{x_{i j}: 1 \leq \mathrm{j} \leq \mathrm{n}\right\}\right)\right\}
$$

and replacing med $_{i}$ in OS by medix, which is the median expression value in normal samples. Further, madi $_{i}$ was replaced by

$$
\operatorname{mad}_{i}=1.4826 \times \operatorname{median}\left(\left\{\left(\mathrm{x}_{i j}-\operatorname{med}_{i x}\right): 1 \leq \mathrm{j} \leq \mathrm{n}\right\},\left\{\left(\mathrm{y}_{i j}-\operatorname{med}_{i y}\right): 1 \leq \mathrm{j} \leq \mathrm{m}\right\}\right) .
$$

where med $_{i y}$ is the median expression value in cancer samples.

COPA and OS statistics were derived from the t-statistic by replacing the mean and standard errors used in the t-statistic with the median and median absolute deviations, respectively. ORT has been proposed as a more robust statistic that utilizes the absolute difference of each expression value from the median instead of the squared difference of each expression value from the average.

\subsubsection{Maximum Ordered Subset t (MOST) statistic}

Lian (2008) argued that OS and ORT statistics used arbitrary outliers and proposed the MOST statistic which consider all possible values for outlier thresholds. The MOST procedure requires cancer sample expression data be sorted in descending order and the following statistic calculated:

$$
\operatorname{MOST}_{i}=\max _{1 \leq \mathrm{k} \leq \mathrm{m}}\left(\frac{\sum_{1 \leq j \leq \mathrm{k}}\left(y_{i j}-\operatorname{med}_{i x}\right)}{\operatorname{mad}_{i}}-\mu_{k}\right) / \delta_{k}
$$

$\mu_{k}$ and $\delta_{k}$ are obtained from the order statistics of $m$ samples generated from a standard normal distribution and are used to make different values of the statistic comparable for different values of $k$. 


\subsubsection{The Least Sum of Ordered Subset Variance t-statistic (LSOSS)}

Wang and Rekaya (2010) proposed a new method named least sum of ordered subset square t-statistic (LSOSS). In LSOSS, mean expression values in normal and cancer samples were considered instead of median expression values. Because if outliers are present among cancer samples, the distribution of gene expression values in cancer samples will have two peaks. The higher peak corresponds to activated samples while the lower peak indicates inactivated samples. Consequently, this outlier issue can be addressed through the idea of detecting a "change point" or "break point" in the ordered gene expression values of the cancer group. A model related to fitting least squares should be effective for this goal. For each gene, an optimal change point in its expression can be detected and could be used to investigate potential outliers in cancer samples. The general idea of LSOSS is to use the sum of squares of two ordered subsets of cancer samples to estimate the square sum of the t-statistic and to use the mean value of the appealing subset of cancer samples to estimate the mean value of cancer samples of the t-statistic.

The LSOSS method involves the following steps:

a) For each gene $i$, the expression levels in cancer samples are sorted in descending order and then divided into two subsets:

$$
S_{i k 1}=\left\{y_{i j}: 1 \leq \mathrm{j} \leq \mathrm{k}\right\}, S_{i k 2}=\left\{y_{i j}: \mathrm{k}+1 \leq \mathrm{j} \leq \mathrm{m}\right\}
$$

b) For the two subsets, the mean and sum of squares for each gene $i$ are calculated:

$$
\begin{gathered}
\bar{y}_{s_{i k 1}}=\operatorname{mean}\left(\left\{y_{i j}: 1 \leq \mathrm{j} \leq \mathrm{k}\right\}\right), \bar{y}_{s_{i k 2}}=\operatorname{mean}\left(\left\{y_{i j}: \mathrm{k}+1 \leq \mathrm{j} \leq \mathrm{m}\right\}\right) \\
S S_{i k 1}=\sum_{1 \leq \mathrm{j} \leq \mathrm{k}}\left(y_{i j}-\bar{y}_{s_{i k 1}}\right)^{2}, S S_{i k 2}=\sum_{\mathrm{k}+1 \leq \mathrm{j} \leq \mathrm{m}}\left(y_{i j}-\bar{y}_{s_{i k 2}}\right)^{2}
\end{gathered}
$$

The only issue left to be solved is the value $k$ that divided the two subsets. For that purpose an exhaustive search was implemented for all possible values ranging from 1 to $m-1$. The 
optimum value of $k$ is obtained by minimizing the pooled sum of squares for cancer samples given by:

$$
\arg \min _{1 \leq \mathrm{k} \leq \mathrm{m}-1}\left(S S_{i k 1}+S S_{i k 2}\right)
$$

Let $S_{i x}^{2}$ be the sum of squares for normal samples given by:

$$
S_{i x}^{2}=\sum_{1 \leq \mathrm{j} \leq \mathrm{n}}\left(x_{i j}-\bar{x}_{i}\right)^{2}
$$

The pooled standard error estimated for gene $i$ is defined by

$$
S_{i}^{2}=\frac{S_{i x}^{2}+S S_{i k 1}+S S_{i k 2}}{n+m-2}
$$

c) The LSOSS statistic for declaring a gene $i$ with outlier differential expression in case samples is computed as:

$$
L S S V_{i}=k \frac{\bar{y}_{s_{i k 1}}-\bar{x}_{i}}{s_{i}}
$$

where $k$ could be interpreted as the number of outlier samples for gene $i$. If repressed gene expression is of interest we can use $L S S V_{i}=(m-k) \frac{\bar{y}_{s_{i k 1}}-\bar{x}_{i}}{s_{i}}$.

\subsection{Proposed Outlier Detection Methods}

We observe that most of the outlier detection techniques defined in the previous section contains some non-robust components such as the mean and standard deviations and consequently they may become ineffective in doing their jobs. In our study we propose three new outlier techniques modifying some of the existing ones. The proposed techniques are described below.

\subsubsection{Expressed Robust t-statistic (ERT)}

We have seen in (4.1) that the two-condition t-statistic for gene $i$ is defined by: 


$$
t_{i}=\frac{\overline{y_{l}}-\overline{x_{l}}}{s_{i}}
$$

Since both $\bar{y}_{l}, \bar{x}_{l}$, and $s_{i}$ are non-robust, we propose the expressed robust t-statistic as:

$$
\grave{t_{i}}=\frac{\operatorname{med}_{\mathrm{iy}}-\mathrm{med}_{\mathrm{ix}}}{\operatorname{mad}_{\mathrm{i}}}
$$

where

$$
\begin{gathered}
\operatorname{med}_{i x}=\operatorname{median}\left(\left\{\mathrm{x}_{i j}: 1 \leq \mathrm{j} \leq \mathrm{n}\right\}\right) . \\
\operatorname{med}_{i y}=\operatorname{median}\left(\left\{\mathrm{y}_{i j}: 1 \leq \mathrm{j} \leq \mathrm{m}\right\}\right) . \\
\operatorname{mad}_{i}=1.4826 \times \operatorname{median}\left(\left\{\left(\mathrm{x}_{i j}-\operatorname{med}_{i x}\right): 1 \leq \mathrm{j} \leq \mathrm{n}\right\},\left\{\left(\mathrm{y}_{i j}-\operatorname{med}_{i y}\right): 1 \leq \mathrm{j} \leq \mathrm{m}\right\}\right) .
\end{gathered}
$$

\subsubsection{Modified Outlier Robust t-statistics (MORT)}

In ORT statistic $R_{i}(4.6)$ is defined as

$$
\mathrm{R}_{i}=\left\{y_{i j}: y_{i j}>\mathrm{q}_{75}\left(\left\{x_{i j}: 1 \leq \mathrm{j} \leq \mathrm{n}\right\}\right)+\operatorname{IQR}\left(\left\{x_{i j}: 1 \leq \mathrm{j} \leq \mathrm{n}\right\}\right)\right\}
$$

We make slight change to define $R_{i}$ to make it equivalent with classical IQR test rule to detect outliers. We define $\mathrm{R}_{\mathrm{i}}$ as

$$
\mathrm{R}_{i}=\left\{y_{i j}: y_{i j}>\mathrm{q}_{75}\left(\left\{x_{i j}: 1 \leq \mathrm{j} \leq \mathrm{n}\right\}\right)+1.5 * \operatorname{IQR}\left(\left\{x_{i j}: 1 \leq \mathrm{j} \leq \mathrm{n}\right\}\right)\right\}
$$

Our modified ORT (MORT) statistic is

$$
\operatorname{MORT}_{i}=\frac{\sum_{y_{i j} \in R_{i}}\left(y_{i j}-\operatorname{med}_{i}\right)}{\operatorname{mad}_{i}}
$$

where $R_{i}$ is defined in (4.15).

\subsubsection{The Least Sum Square of Ordered Subset Robust t-statistic (LSOSRT)}

The general idea of least sum square of ordered subset robust t-statistic (LSOSRT) is to use the sum of squares of two ordered subsets of cancer samples to estimate the square sum of 
the t-statistic and to use the median value of the appealing subset of cancer samples to estimate the median value of cancer samples of the t-statistic. Since we have few non-robust components in it, we need to replace those components. The proposed LSOSRT method involves the following steps:

a) For each gene $i$, the expression levels in cancer samples are sorted in descending order and then divided into two subsets:

$$
S_{i k 1}=\left\{y_{i j}: 1 \leq \mathrm{j} \leq \mathrm{k}\right\}, S_{i k 2}=\left\{y_{i j}: \mathrm{k}+1 \leq \mathrm{j} \leq \mathrm{m}\right\}
$$

b) For the two subsets, the mean and sum of squares for each gene i are calculated:

$$
\begin{gathered}
\bar{y}_{s_{i k 1}}=\operatorname{mean}\left(\left\{y_{i j}: 1 \leq \mathrm{j} \leq \mathrm{k}\right\}\right), \bar{y}_{s_{i k 2}}=\operatorname{mean}\left(\left\{y_{i j}: \mathrm{k}+1 \leq \mathrm{j} \leq \mathrm{m}\right\}\right) \\
S S_{i k 1}=\sum_{1 \leq \mathrm{j} \leq \mathrm{k}}\left(y_{i j}-\bar{y}_{s_{i k 1}}\right)^{2}, S S_{i k 2}=\sum_{\mathrm{k}+1 \leq \mathrm{j} \leq \mathrm{m}}\left(y_{i j}-\bar{y}_{s_{i k 2}}\right)^{2}
\end{gathered}
$$

The only issue left to be solved is the value $k$ that divided the two subsets. For that purpose an exhaustive search was implemented for all possible values ranging from 1 to $m-1$. The optimum value of $k$ is obtained by minimizing the pooled sum of squares for cancer samples given by:

$$
\arg \min _{1 \leq \mathrm{k} \leq \mathrm{m}-1}\left(S S_{i k 1}+S S_{i k 2}\right)
$$

The pooled mad estimated for gene $i$ is defined by

$$
\begin{aligned}
& \operatorname{mad}_{i}^{p}=1.4826 \times \operatorname{median}\left(\left\{\left(\mathrm{x}_{i j}-\operatorname{med}_{i x}\right): 1 \leq \mathrm{j} \leq \mathrm{n}\right\},\left\{\left(\mathrm{y}_{i j}-\operatorname{med}_{i y}\right): 1 \leq \mathrm{j} \leq\right.\right. \\
& \left.\mathrm{k}\},\},\left\{\left(\mathrm{y}_{i j}-\operatorname{med}_{i y}\right): \mathrm{k}+1 \leq \mathrm{j} \leq \mathrm{m}\right\}\right) .
\end{aligned}
$$

c) The LSOSS statistic for declaring a gene $i$ with outlier differential expression in case samples is computed as:

$$
\operatorname{LSSV}_{i}=k \frac{\operatorname{med}_{i y}\left\{y_{i j}: 1 \leq \mathrm{j} \leq \mathrm{k}\right\}-\operatorname{med}_{i x}\left\{x_{i j}: 1 \leq \mathrm{j} \leq \mathrm{n}\right\}}{\operatorname{mad}_{i}^{p}}
$$

where $k$ could be interpreted as the number of outlier samples for gene $i$. 


\subsection{Monte Carlo Comparison of Different Outlier Detection Methods}

Before applying to real data we tested the performance of our newly proposed data by simulation studies. Simulation studies were conducted to compare the performance of newly proposed three methods ERT, MORT and LSOSRT with the t-statistic, COPA, OS, ORT, MOST and LSOSS. The simulation was conducted in different situations. To test and check the consistency of the test statistic, we generate gene expression for two groups of sample with different sizes in different simulation.

Table 4.1: Outliers in different methods in Simulation Study

\begin{tabular}{|l|c|c|c|c|c|}
\hline & $n=75 m=25$ & $n=60 m=40$ & $n=55 m=45$ & $n=80 m=20$ & $n=90 m=10$ \\
\hline T & 20 & 13 & $g=40$ & $g=40$ & $g=40$ \\
\hline COPA & $\mathbf{6}$ & $\mathbf{5}$ & $\mathbf{1 5}$ & $\mathbf{4}$ & $\mathbf{5}$ \\
\hline OS & $\mathbf{1 7}$ & $\mathbf{1 5}$ & $\mathbf{9}$ & $\mathbf{6}$ & $\mathbf{2}$ \\
\hline ORT & 7 & $\mathbf{5}$ & $\mathbf{5}$ & 2 & $\mathbf{4}$ \\
\hline LSSOS & $\mathbf{3}$ & $\mathbf{0}$ & $\mathbf{0}$ & $\mathbf{0}$ & $\mathbf{0}$ \\
\hline ERT & $\mathbf{1 9}$ & $\mathbf{2 0}$ & $\mathbf{2 0}$ & $\mathbf{2 0}$ & $\mathbf{2 0}$ \\
\hline MORT & $\mathbf{1 2}$ & $\mathbf{6}$ & $\mathbf{6}$ & $\mathbf{5}$ & $\mathbf{4}$ \\
\hline LSOSRT & $\mathbf{1 2}$ & $\mathbf{3}$ & $\mathbf{5}$ & $\mathbf{1 5}$ & $\mathbf{8}$ \\
\hline
\end{tabular}

In all simulation we generated $g=40$ genes. Out of 40 genes we generated 20 genes considering no differences between normal and tumor group. We generated these 20 genes with uniform condition for both groups. Further, we generated another 20 genes with two different situations. To distinguish the two groups, for normal sample and tumor sample we used different 
ranges. We assume outliers do exist in later 20 genes. The process is done 5 times by changing the number of normal and tumor sample sizes. For the first set of simulation we generated $n=75$ and $m=25$ as number of samples from normal and tumor group respectively. For other simulations we chose $(n=60, m=40),(n=55, m=45),(n=80, m=20)$ and $(n=90, m=10)$. We applied all the existing methods and our new methods to these simulated data. The results of the number of genes detected as outliers in this simulation experiment are given in Table 4.1.

Results presented in Table 4.1 show that among the existing methods, the $\mathrm{T}$ test performs well but on a couple of occasions it fail to identify the genuine outliers. The performances of COPA, OS and ORT are not very satisfactory. But the performance of LSSOS is the worst. Most of the times it fails to identify even a single outlier. All newly proposed methods give the better result comparing to the existing corresponding method, however, the ERT performs the best. The methods give consistent results over different simulations.

\subsection{Comparison of Different Outlier Detection Methods for Colon Cancer}

\section{Data}

Now we apply the outlier detection methods in cancer data. In the study data we have the data set of intensities of 2,000 genes in 22 normal and 40 tumor colon tissues. The genes chosen are the 2,000 genes with highest minimal intensity across the samples. We try to find out the possible responsible genes for tumor. We believe genes with high expressions might be guilty for tumor. Considering this we applied the existing outlier detection methods and also the newly proposed three methods to find out the possible genes. 

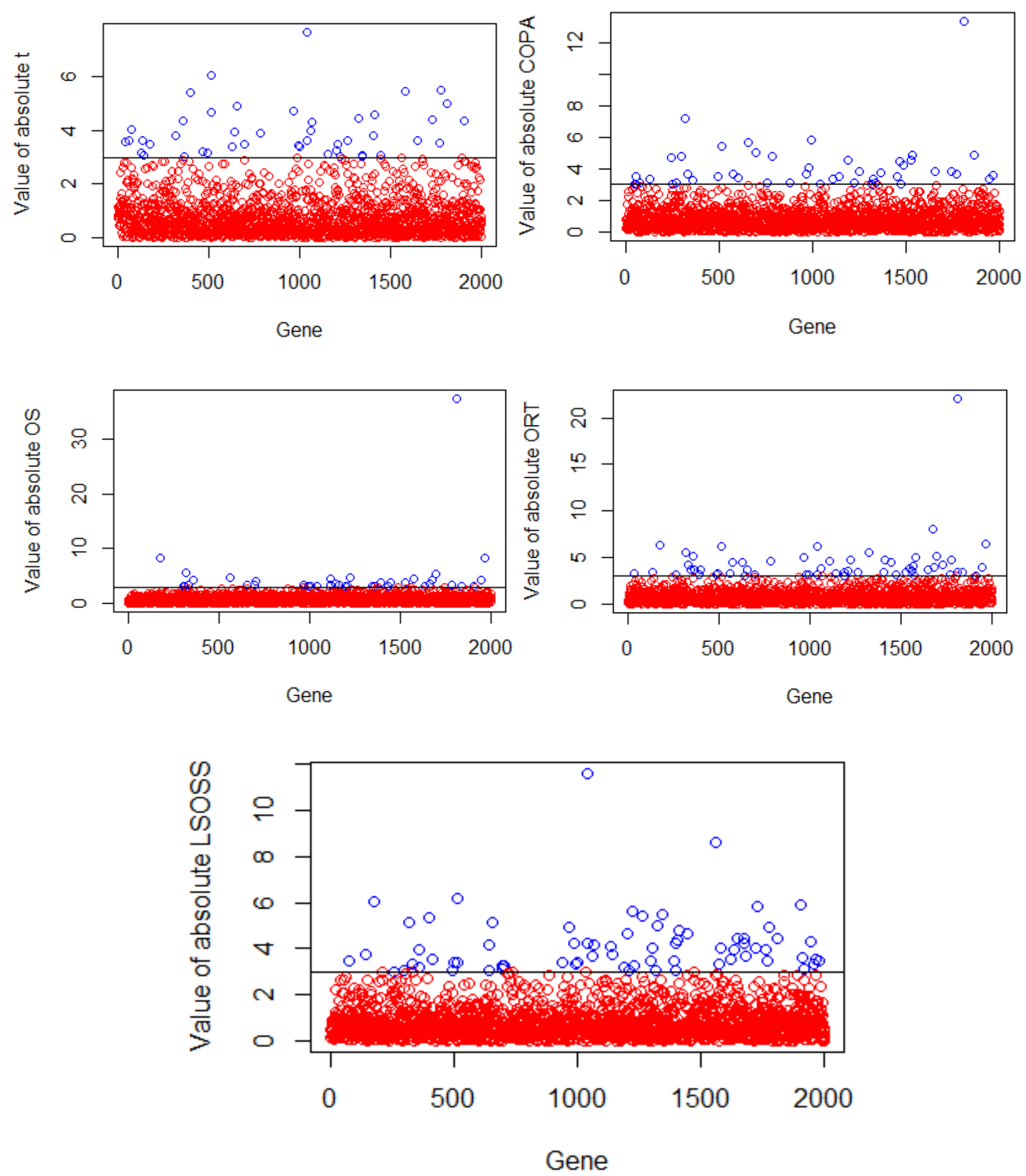

Figure 4.1: Index Plot of Genes with Cut-off Points for Existing Outlier Detection Methods 

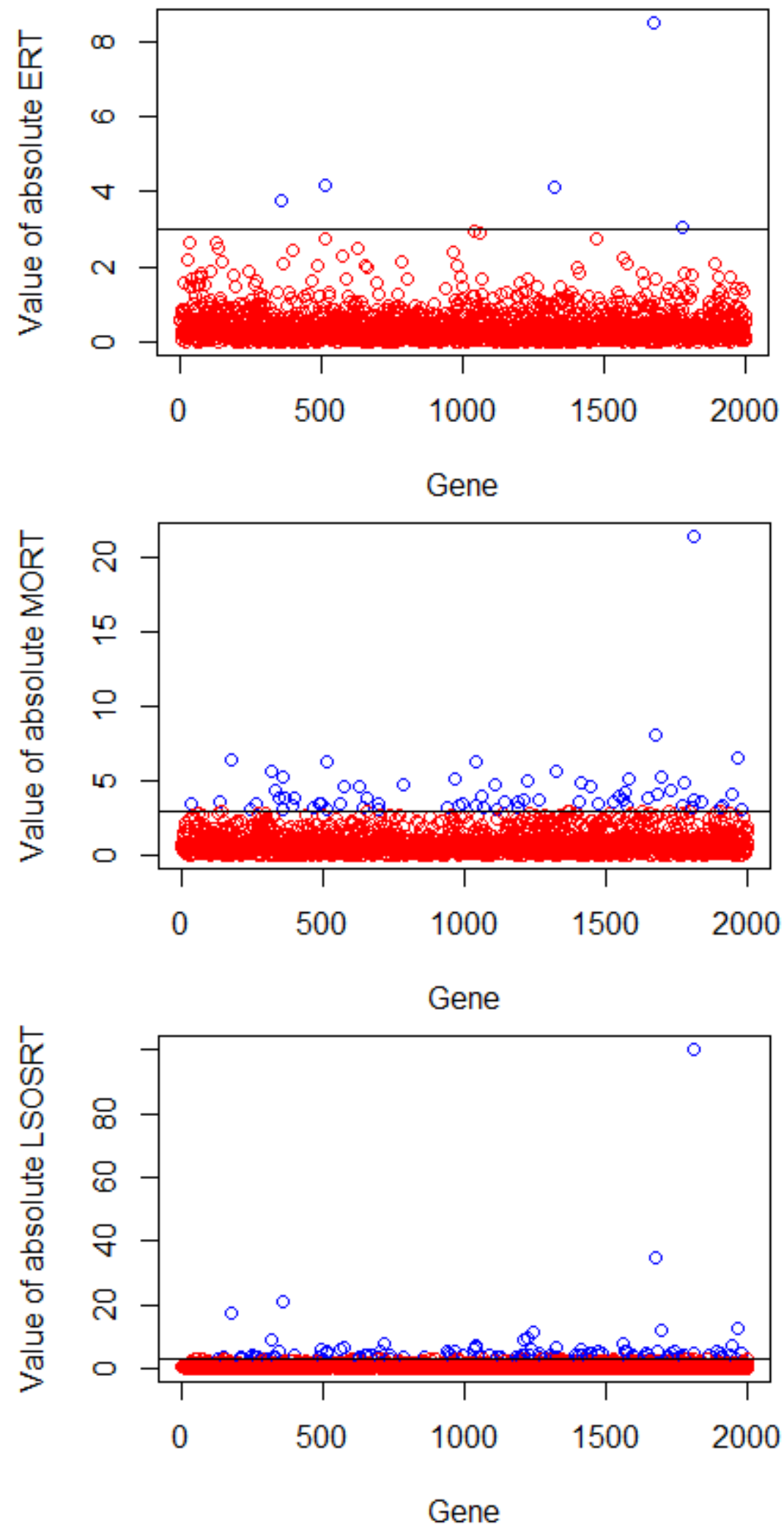

Figure 4.2: Index Plot of Genes with Cut-off Points for Proposed Outlier Detection Methods 
Figures 4.1 and 4.2 give a graphical display of the performances of the existing and proposed outluier detection methods with their respective cut-off points. The Table 4.2 demonstrates the number of outliers detected by different methods.

Table 4.2: Number of Detected Outliers for Colon Cancer Data

\begin{tabular}{|c|c|}
\hline Method & $\begin{array}{c}\text { Number of Outliers } \\
(n=22, m=40, g=2000)\end{array}$ \\
\hline t-test & 46 \\
\hline COPA & 49 \\
\hline OS & 38 \\
\hline ORT & 58 \\
\hline LSSOS & 72 \\
\hline ERT & 5 \\
\hline MORT & 69 \\
\hline LSOSRT & 118 \\
\hline
\end{tabular}

The above figures and table show that the traditional t-test can successfully identify 46 genes as outliers. The COPA, OS, ORT, MOST and LSSOS statistic identify 49, 38, 58 and 78 genes respectively. In turn, our newly proposed statistic ERT, MORT and LSOSRT can identify 5, 69 and 118 genes. 


\section{CHAPTER 5}

\section{CONCLUSIONS AND AREAS OF FUTURE RESEARCH}

In this chapter we will summarize the findings of our research to draw some conclusions and outline ideas for our future research.

\subsection{Conclusions}

In our study we propose several new outlier detection techniques for finding outliers in gene expression data. The simulation results suggest that performances of proposed statistic are better than existing methods. When evaluating ERT, MORT and LSOSRT based on tumor cancer data, we studied how many genes among the 2000 genes selected separately by different statistical approaches. The numbers of tumor cancer related genes identified by existing methods were 46, 49, 38, 58, and 78 for the t-statistics, COPA, OS, ORT and MOST respectively. However, our proposed methods ERT, MORT and LSOSRT have identified 5, 69 and 118 tumor cancer related genes. Disentanglement the heterogeneous designs of cancer samples is an important goal in medical research, especially for clinical diagnosis and the molecular understanding of cancer mechanisms. The diverse patterns of oncogene activation have been well studied and several useful statistical tools have been proposed. ERT, MORT and LSOSRT are reasonable model to detect cancer outlier differential gene expression.

For each gene, ERT distinguish the expression values of normal and tumor samples. If any gene is expressed heterogeneously in cancer samples, the mean and variance of gene 
expression values in cancer samples are overemphasized by the classical t-statistic while ERT used the robust statistic Median and MAD in replace of them which gives a reasonable estimate.

MORT tries to find a region which might be more appropriate for the outliers to be located in. MORT uses classical approach of IQR to detect outlier. The only improvisation in MORT compare to existing ORT is that MORT consider the distance of $\left(\mathrm{Q}_{3}+1.5\right.$ time of IQR) to suspect outlier. Thus MORT is safer estimate to detect cancer outliers more easily.

Like LSOSS, LSOSRT tries to find an optimal "change point" in the ordered expression values of cancer samples. Furthermore, LSOSS uses the mean value of the appealing subset instead of the overall mean value of the cancer samples while LSOSRT uses median value which is more robust. Thus, LSOSRT detects cancer outliers more easily. If one gene is expressed homogeneously in cancer samples, LSOSRT still works well because it uses robust statistic similarly to the ERT-statistic.

Our proposed scheme could be useful tool to separate the patterns of tumor cancer with specific gene signatures. In addition, these heterogeneous gene activation patterns may be regarded as the signatures for subtypes of tumor cancer. Thus, the procedure presented could also be useful in detecting and classifying tumor cancer subtypes. Our approach, however, differed from previous studies mainly in that the classification is based on different combinational activation patterns of candidate genes instead of clustering their expression values.

\subsection{Areas of Future Research}

In our research, mainly because of time constraints, we could not look at the different aspects of outlier detection. In our simulation we consider normal and tumor data both of which come from normal distribution. But gene expression data like RNA-seq data are count data. So 
we need to investigate the performance of the proposed methods on that type of observations. Now a days there is a growing demand of nonparametric outlier detection methods such as bootstrap outlier (bootlier) detection method. We would like to develop nonparametric methods for the identification of outliers in gene expression data. 


\section{REFERENCES}

Acuna E., Rodriguez C. A., "Meta analysis study of outlier detection methods in classification," Technical paper, Department of Mathematics, University of Puerto Rico at Mayaguez, Retrived from academic.uprm.edu/ eacuna/paperout.pdf. In proceedings IPSI 2004, Venice, 2004.

Barnett V., Lewis T. (1994) “Outliers in Statistical Data”. John Wiley, 1994.

Fawcett T., Provost F. (1997) "Adaptive fraud detection," Data-mining andKnowledge Discovery, 1(3), 291-316, 1997.

Grubbs, Frank E. (1950) Sample criteria for testing outlying observations, The Annals of Mathematical Statistics 21(1), p.27-58, doi: 10.1214/aoms/1177729885, March 1950

Hadi, A.S., Imon, A.H.M.R. and Werner, M. (2009). Detection of outliers, Wiley Interdisciplinary Reviews: Computational Statistics, 1, pp. $57-70$.

Hampel, F.R. (1971) A general qualitative definition of robustness, Annals of mathematics Statistics, 42, (1971), 1887-1896.

Hampel, F.R., Ronchetti, E.M., Rousseeuw, P.J. and Stahel, W. (1986). Robust Statistics: The Approach Based on Influence Function, Wiley, New York.

Hawkins D. (1980), Identification of Outliers, Chapman and Hall, 1980.

Johnson T., Kwok I., Ng R., "Fast Computation of 2-Dimensional Depth Contours," In Proceedings of the Fourth International Conference on Knowledge Discovery and Data Mining, 224-228. AAAI Press, 1998.

Lian H. (2008) MOST: detecting cancer differential gene expression. Biostatistics. 2008;9:4118. 
Liu H., Shah S., Jiang W., "On-line outlier detection and data cleaning," Computers and Chemical Engineering, 28, 1635-1647, 2004.

Penny K. I., Jolliffe I. T., "A comparison of multivariate outlier detection methods for clinical laboratory safety data," The Statistician 50(3), 295-308, 2001.

R. B. Dean and W. J. Dixon (1951) "Simplified Statistics for Small Numbers of Observations". Anal. Chem., 1951, 23 (4), 636-638.

Ruts I., Rousseeuw P. (1996) "Computing Depth Contours of Bivariate Point Clouds, " In Computational Statistics and Data Analysis, 23,153-168, 1996.

Tibshirani R, Hastie T. (2007) Outlier sums for differential gene expression analysis. Biostatistics. 2007;8:2-8.

Tomlins SA, Rhodes DR, Perner S, et al. (2005) Recurrent fusion of TMPRSS2 and ETS transcription factor genes in prostate cancer. Science. 2005; 310:644-8.

U. Alon, N. Barkai, D. A. Notterman, K. Gish, S. Ybarra, D. Mack, and A. J. Levine (1999) "Broad patterns of gene expression revealed by clustering of tumor and normal colon tissues probed by oligonucleotide arrays" published in the Proceedings of National Academy Science. USA, Vol. 96, Issue 12, 6745-6750, June 8, 1999.

Wang Y and Rekaya R LSOSS: Detection of Cancer Outlier Differential Gene Expression, Biomarker Insights 2010:5 69-78

Wu B. (2007) Cancer outlier differential gene expression detection. Biostatistics. 2007;8:56675. 


\section{APPENDIX}

\section{Sample data ( $1^{\text {st }}$ Four Genes with all samples)}

Gene

$\begin{array}{ll}\text { Hsa.3004 } & 9164.25 \\ \text { Hsa.13491 } & 6719.53 \\ \text { Hsa.13491.1 } & 4883.45 \\ \text { Hsa.37254 } & 3718.16 \\ & \\ \text { Hsa.3004 } & 4865.22 \\ \text { Hsa.13491 } & 3237.78 \\ \text { Hsa.13491.1 } & 2341.09 \\ \text { Hsa.37254 } & 2372.68 \\ & \\ \text { Hsa.3004 } & 7121.17 \\ \text { Hsa.13491 } & 5346.65 \\ \text { Hsa.13491.1 } & 4254.12 \\ \text { Hsa.37254 } & 3399.71\end{array}$

$$
\begin{aligned}
& \text { Hsa } \\
& \text { Hsa } \\
& \text { Hsa }
\end{aligned}
$$

$\begin{array}{lrrrrrrrr}\text { Hsa.3004 } & 7144.41 & 5382.39 & 7434.82 & 4214.9 & 8865.46 & 5934.89 & 5821.62 & 9767.03 \\ \text { Hsa.13491 } & 2071.4 & 3848.44 & 6471.21 & 2213.36 & 5447.19 & 3744.99 & 3748.25 & 9785.78 \\ \text { Hsa.13491.1 } & 1619.28 & 3372.49 & 5029.62 & 1611.52 & 4887.06 & 3528.83 & 3439.95 & 8605.04 \\ \text { Hsa.37254 } & 2576.16 & 4444.57 & 4749.33 & 1764.23 & 3782.59 & 2721.7 & 2502.97 & 4941.24 \\ & & & & & & & & \\ \text { Hsa.3004 } & 13324.73 & 12977.71 & 8753.24 & 5012.02 & 6904.8 & 8347.98 & 5100.54 & 4554.58 \\ \text { Hsa.13491 } & 9505.03 & 7565.62 & 8978.13 & 1383.49 & 2260.78 & 9852.3 & 3343.32 & 3139.31 \\ \text { Hsa.13491.1 } & 7740.99 & 5735.2 & 7777.84 & 1269.65 & 1987 & 8178.97 & 2925.67 & 2958.4 \\ \text { Hsa.37254 } & 7982.81 & 6911.68 & 6202.5 & 3168.71 & 3439.41 & 4467.5 & 2914.19 & 1658.31 \\ & & & & & & & & \\ \text { Hsa.3004 } & 5466.93 & 4201.51 & 9128.12 & 3799.09 & 14641.93 & 9443.88 & 14368.28 & 6951.35 \\ \text { Hsa.13491 } & 10152.27 & 2425.63 & 5502.72 & 5665.78 & 3377.39 & 6671.78 & 3651.99 & 5565.78 \\ \text { Hsa.13491.1 } & 7760.92 & 2228.82 & 4590.13 & 5157.07 & 3048.43 & 6260.62 & 3302.93 & 4480.79 \\ \text { Hsa.37254 } & 7258.25 & 1503.61 & 3234.4 & 1359.79 & 5401.23 & 4905.71 & 5738.49 & 3667.65 \\ & & & & & & & & \\ \text { Hsa.3004 } & 6357.92 & 11605.97 & 7666.68 & 4527 & 4653.24 & 4972.17 & 9112.37 & 6234.62 \\ \text { Hsa.13491 } & 3736.85 & 8278.92 & 6409.74 & 2912.31 & 6251.56 & 4173.92 & 6824.49 & 4005.3 \\ \text { Hsa.13491.1 } & 2665.59 & 7316.1 & 6489.86 & 2124.99 & 4947.12 & 3668.53 & 5982.85 & 3093.68 \\ \text { Hsa.37254 } & 2493.88 & 3602.35 & 2451.12 & 2609.05 & 3763.55 & 1567.56 & 3147.04 & 3183.09\end{array}$

Intensities from 22 Normal Tissue Samples

$\begin{array}{lllll}6246.45 & 2510.33 & 4028.71 & 5271.52 & 14173.05\end{array}$

7823.53

5955.84

3975.56

1960.65

4740.77

3318.51

8411.86

6042.84

8766.05

$4985.22 \quad 5627.25$

$4735.79 \quad 3619.53$

$4075.12 \quad 2606.5$

$2845.25 \quad 2544.45$

4412.48

1752.4

$6995.41 \quad 1914.68$

3656.78

6194.25

11447.63

6302.78

1280.32

5200.34

6658.11

5082.86

5202

3529.13

4127.5

$\begin{array}{lll}5316.4 & 1595.41 & 4101.92\end{array}$

4079.63

$2920.84 \quad 3635.7$

1365.7

3951.2

$5445.86 \quad 2604.74$

6870.32

4751.76

4177.28

5777.17

6730.63

7472.01

$4984.1 \quad 3387.89$

3472.13

3653.93

$4585.9 \quad 2413.65$

2559.46

2728.22

3182

$3165.16 \quad 2843.26$

2624.69

3494.48

Intensities from 40 Tumor Tissue Samples

Hsa.13491

8589.42

3825.71

3230.33

7126.6

9330.68

14876.41

4469.09

4913.8

6970.36

3694.45

3779.07

7017.23

3201.9

5167.06

5215.05

5369.97

3400.74

4723.78

2327.63

4773.68

4288.62

4263.41

4705.65

3463.59

(3705.55

9491.53

11248.68

3330.36

3213.43 


\section{R Code}

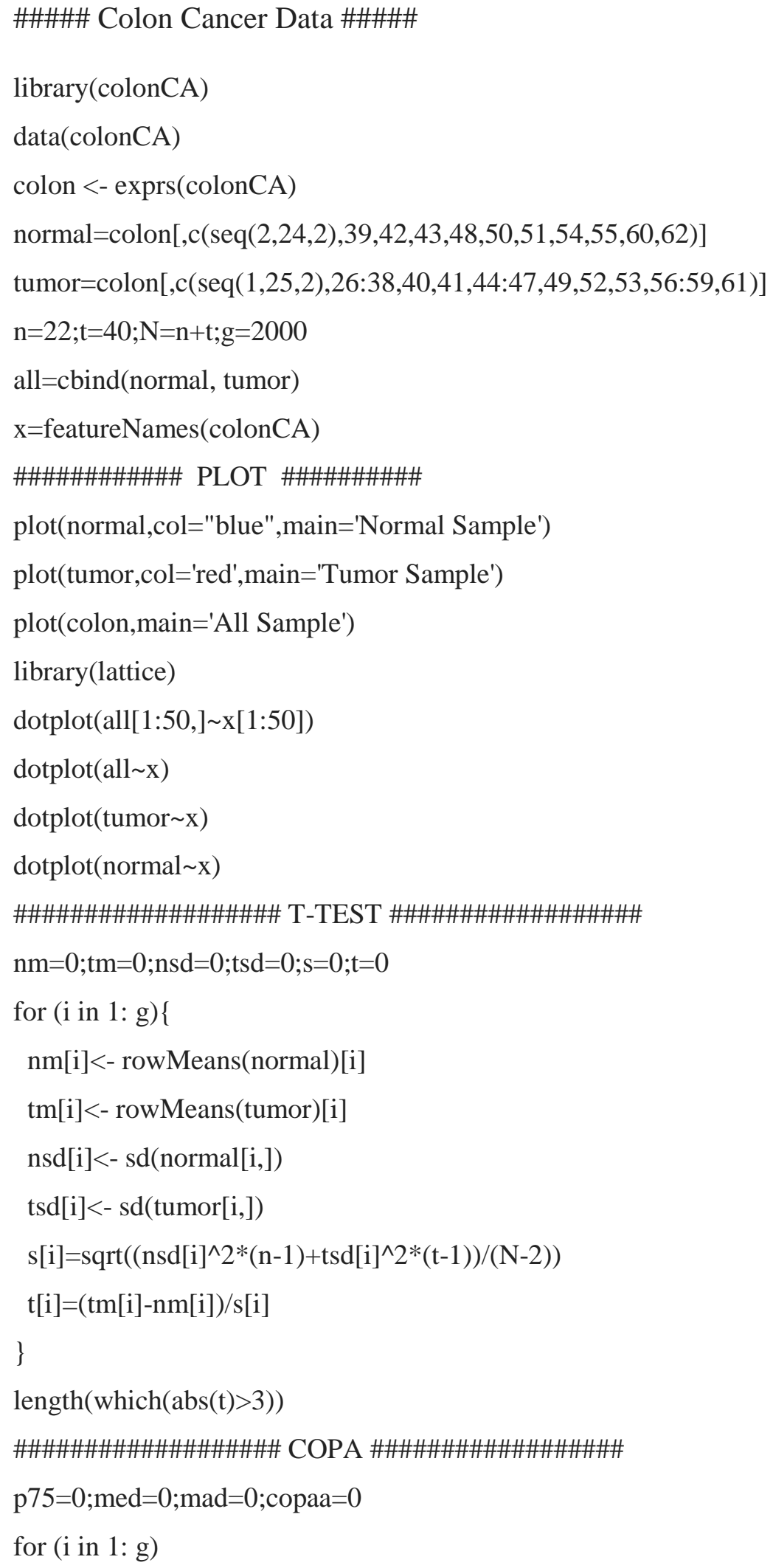




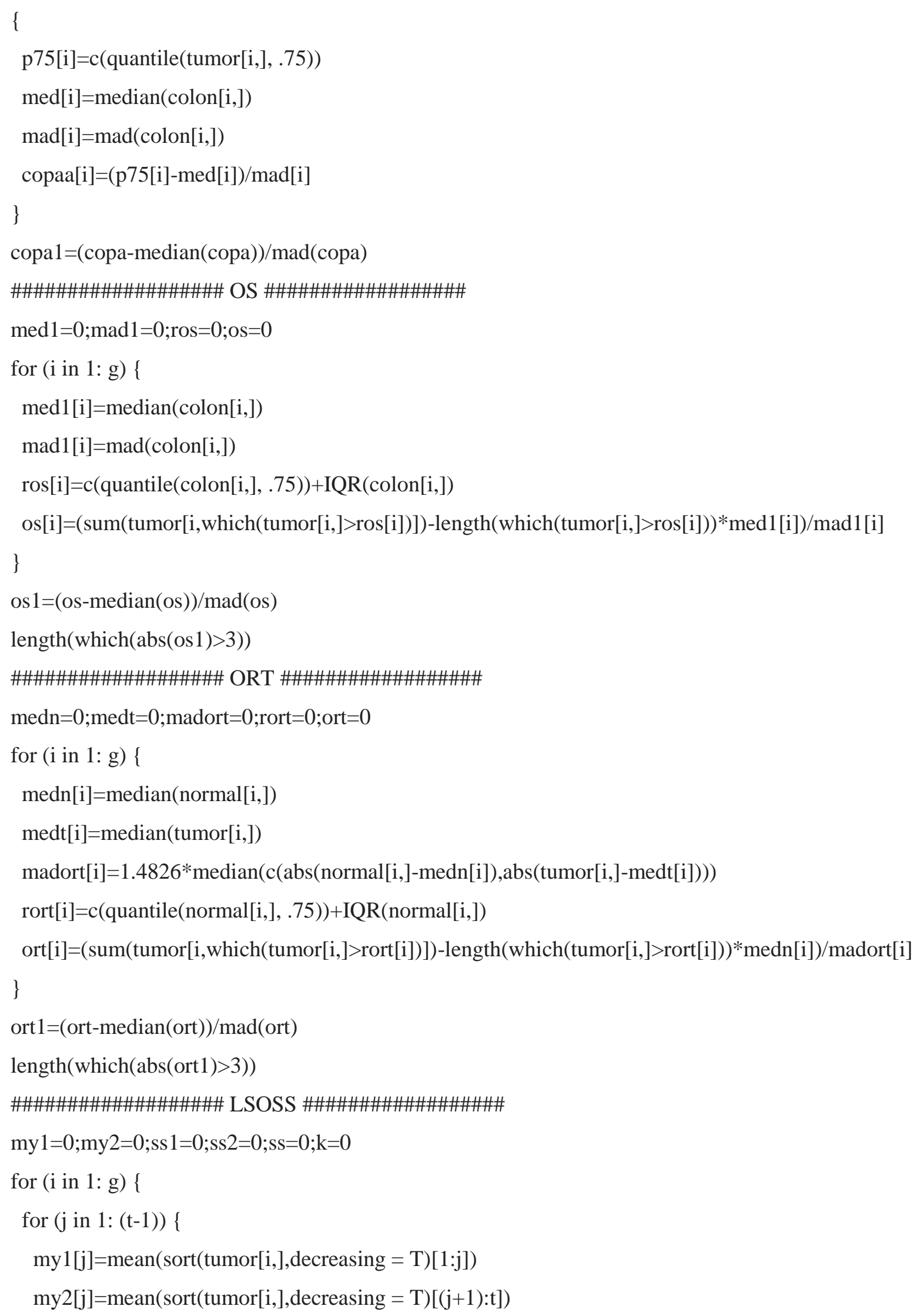




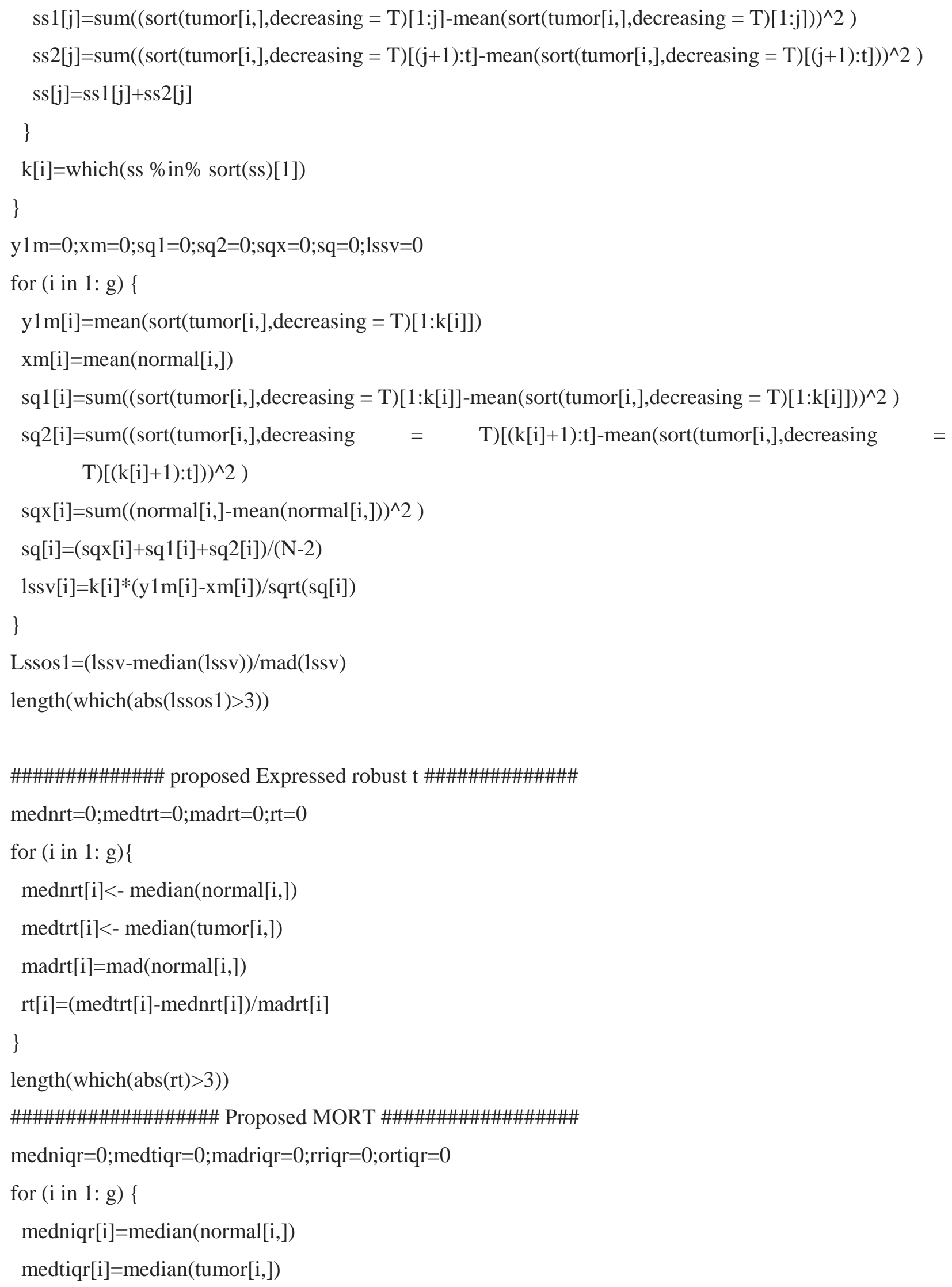




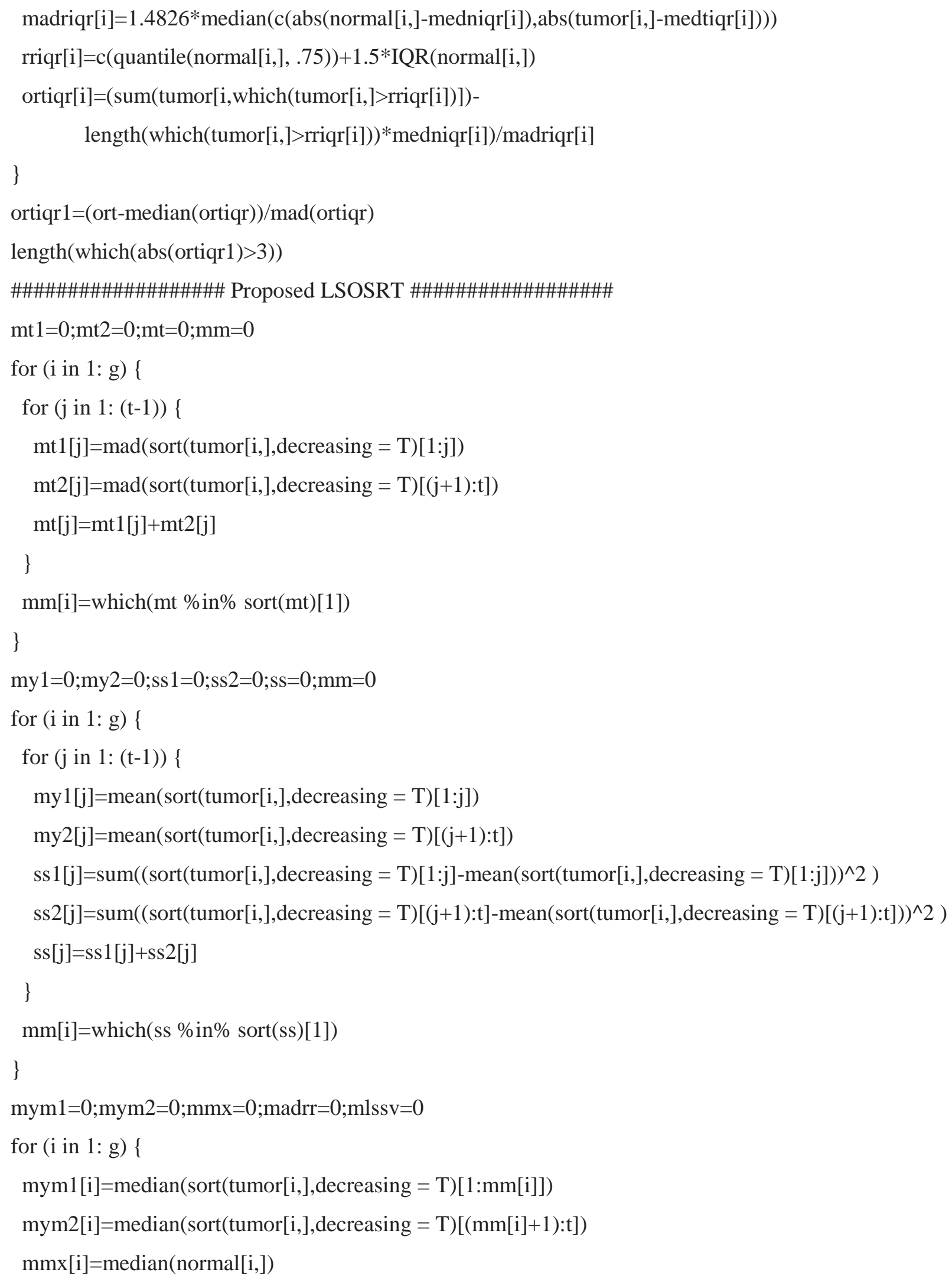




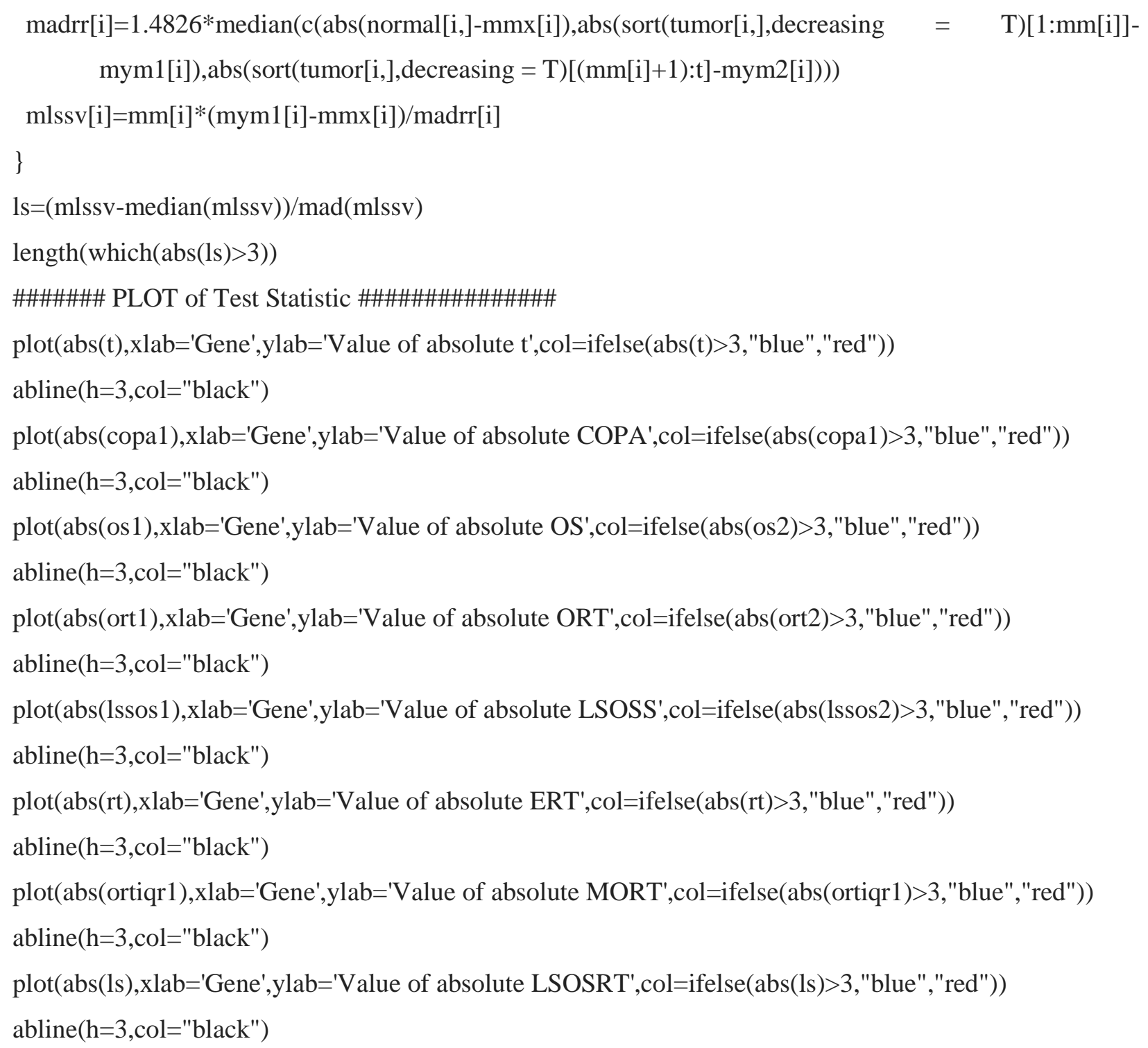

